
\title{
$\begin{array}{ll}\text { Research Square } & \begin{array}{l}\text { Preprints are preliminary reports that have not undergone peer review. } \\ \text { They should not be considered conclusive, used to inform clinical practice, } \\ \text { or referenced by the media as validated information. }\end{array}\end{array}$
}

\section{Aging and MPTP-Sensitivity Depend on Molecular and Ultrastructural Signatures of Astroglia and Microglia in Mice Substantia Nigra}

\author{
PL Abhilash \\ National Institute of Mental Health and Neuro Sciences \\ Upasna Bharti \\ National Institute of Mental Health and Neuro Sciences \\ Mariamma Philip \\ National Institute of Mental Health and Neuro Sciences \\ Santhosh Kumar Rashmi \\ National Institute of Mental Health and Neuro Sciences \\ Trichur R Raju \\ National Institute of Mental Health and Neuroscience \\ Bindu M Kutty \\ National Institute of Mental Health and Neuro Sciences \\ BK Chandrasekhar Sagar \\ National Institute of Mental Health and Neuro Sciences \\ Phalguni Anand Alladi ( $\sim$ alladiphalguni@yahoo.com ) \\ National Institute of Mental Health and Neuro Sciences https://orcid.org/0000-0002-2876-3478
}

\section{Research}

Keywords: Parkinson's disease, 1-methyl-4-phenyl-1, 2, 3, 6-tetrahydropyridine (MPTP), Cytokine ELISA, Neuroinflammation, Monoamine oxidases A\&B, Unbiased stereology, C57BL/6J, CD-1 white mice, substantia nigra pars compacta, Fecal microbiome

Posted Date: February 12th, 2021

DOl: https://doi.org/10.21203/rs.3.rs-190412/v1

License: @ (i) This work is licensed under a Creative Commons Attribution 4.0 International License. Read Full License 


\section{Abstract}

Background

Both astroglia and microglia show region-specific distribution pattern in the central nervous system and often maladapt to age-associated alterations within their niche. Studies on autopsied substantia nigra of Parkinson's disease (PD) patients and experimental models propose gliosis as a trigger for neuronal loss. Epidemiological studies propose an ethnic bias in PD prevalence, since Caucasians are more susceptible than non-whites living in Asia and Africa. Similarly, different mice strains are variably sensitive to the neurotoxin MPTP (1-methyl-4-phenyl-1,2,3,6-tetrahydropyridine). We had earlier likened divergent MPTPsensitivity of C57BL/6J and CD-1 mice with differential susceptibility to PD, based on differences in neuronal numbers.

Methods

Here we examined whether the variable susceptibility was also incumbent to inter-strain differences in the glial features in the substantia nigra pars compacta $(\mathrm{SNpc})$ of C57BL/6J and CD-1 mice. We performed unbiased stereology to quantify iba-1 immunoreactive microglia and s100 $\beta$ immunopositive astroglia on immunohistochemically stained sections. Further, ELISA based estimation of pro-inflammatory and anti-inflammatory cytokines was supplemented with estimation of enzymes like fractalkine, hemeoxygenase, and monoamine oxidases A and B. Electron microscopy was performed to compare the effects on the organelles.

Results

Stereological counts showed more microglia and fewer astrocytes in the substantia nigra of MPTP-susceptible normal C57BL/6J mice, which suggests persistence of an immune-vigilant state. MPTP caused induction of microgliosis and astrogliosis in both strains, suggesting the involvement of these cells in pathogenesis. ELISA of pro-inflammatory cytokines in the ventral-midbrain revealed augmentation of TNF- $a$ and IL- 6 at middle-age in both strains that reduced at old-age, suggesting middle-age as a critical, inflamm-aging associated time-point. TNF-a levels were persistently high in C57BL/6J, through aging and postMPTP; while IL- 6 and IL-1 $\beta$ were upregulated at old-age. CD-1 had higher levels of anti-inflammatory cytokine TGF- $\beta$. MPTP-challenge caused upregulation of enzymes MAO-A, MAO-B and iNOS in both strains. Post-MPTP enhancement in fractalkine and hemeoxygenase-1, may be neuronal compensatory signals. Lastly, ultrastructural observations of elongated mitochondria in astroglia and microglia vis-à-vis the shrunken ones in neurons, suggest upscaling of their functions with neurotoxic consequences.

Conclusions

Thus, astroglia and microglia play a critical role in modulating aging and the susceptibility of an individual to PD.

\section{Highlights}

- CD-1 substantia nigra has higher number of astroglia and fewer microglia than C57BL/6J

- Both mice show age and MPTP-induced gliosis in the substantia nigra pars compacta

- CD-1 nigra has lower levels of pro- and higher levels of anti-inflammatory cytokines

- Tilt of balance between pro- and anti-inflammatory cytokines begins at middle age

- Astrocytes and microglia show elongated mitochondria and intact ER upon MPTP-injection

\section{Introduction}

Inflamm-aging refers to the alterations in the neuron-glia communication during aging, that result from a persistent functional decline in the immune system, characterized by a generalized increase in the pro-inflammatory markers (Franceschi et al., 2006). The system copes by releasing anti-inflammatory cytokines; a process termed as "anti inflamm-aging". The imbalance between inflamm-aging and anti inflamm-aging associated processes, supported by the genetic make-up of the individual and environmental factors combine to trigger the onset or protect against age-related neurodegenerative diseases like Parkinson's disease (PD). PD is characterized by a selective loss of dopaminergic (DA) neurons, primarily in the substantia nigra pars compacta (SNpc), resulting in striatal dopamine depletion as well as dysfunction of the basal ganglia circuitry (Bernheimer et al., 1973; Damier et al., 1999). Non-motor symptoms like constipation, precede the motor symptoms by several years, thus suggesting a role for gut microbiota in the disease pathogenesis (Chaudhuri et al., 2006).

PD is characterized by T-cell infiltration, microgliosis and astrogliosis (McGeer et al., 1988; Kohutnicka et al., 1998). Several preclinical and epidemiological studies point at chronic neuroinflammation as a prototypical event preceding and accompanying neuronal dysfunction. Neuroinflammation marks the presence of activated microglia and reactive astrocytes, direct participation of the adaptive immune system as well as increased synthesis of cytokines, chemokines, inflammatory markers, reactive oxygen and nitrogen species (Boje and Arora, 1992; Boka et al., 1994; Kim et al., 2000). Midbrain DA neurons express the receptors for cytokines such as tumor necrosis factor (TNF- $\alpha$ ), interleukin-1 $\beta$ (IL-1 $\beta$ ) and interferon- $\gamma$ (IFN- $\gamma)$, pointing at their sensitivity to these cytokines (Boka et al., 1994; Mogi et al., 1994).

Epidemiological studies on PD suggest prevalence and incidence rates of approximately 108-257/100,000 and 11-19/100,000 person year, respectively (Van Den Eeden et al., 2003) in Europe. North America reported 329 - 107/100,000 in Nebraska-USA (Strickland and Bertoni, 2004) and 224 per 100,000 personyears in persons above 65 years (Wirdefeldt et al., 2011). The incidence among Indians, Chinese and Malays were less compared to the Westerners (GourieDevi M 2014; Abbas et al., 2017). Thus, the white populations have significantly higher prevalence than non-whites; the mechanisms for which are unclear. We demonstrated the role of nreservation of niaral neurnns with age, maintenance of GNDF receptors and non-logarithmic increase in a-synuclein as few Loading [MathJax]/jax/output/CommonHTML/fonts/TeX/fontdata.js

Page $2 / 24$ 
neuroprotective factors in Asian Indians (Alladi et al., 2009; Alladi et al., 2010 a\&b). We also found age-related morphological transformation of astrocytes and microglia (Jyothi et al., 2016). Direct comparisons on human tissues between populations were not possible.

A reliable recapitulation of the PD pathology in an animal model is observed following the injection of the neurotoxin 1-methyl-4-phenyl-1, 2, 3, 6tetrahydropyridine (MPTP) (Jackson-Lewis and Przedborski, 2007). Different mice strains show varying responses to MPTP, for example, C57BL/6J mice is more sensitive while CD-1 white, BALBc and Swiss Webster are resistant (Smeyne et al., 2001). C57BL/6J DA neurons exposed to MPP ${ }^{+}$(1-methyl-4phenylpyridinium) demonstrated a $39 \%$ loss when cultured on C57BL/6J glia compared to $17 \%$ neuron loss when cultured on SWR/J glia. Thus glia may modulate the strain-dependent susceptibility of mice to MPTP and MPP ${ }^{+}$.

We have earlier reported that the CD-1 had more substantia nigra DA neurons than C57BL/6J and were better protected against MPTP (Vidyadhara et al., 2017). Soreq et al., (2017) reported that, astroglia show significant senescence-associated changes in gene expression patterns. Yet, the role of glia in disease modulation as also in differential vulnerability is not completely understood. In the present study we systematically investigated the responses of astroglia and microglia in the substantia nigra of the two different mice strains i.e. C57BL/6J and CD-1, in terms of aging and inflammatory responses to MPTP. Studies in the last decade suggest a correlation between the gut microbiome and maturation as well as activation of the microglia (Heijtz et al., 2011; Erny et al., 2015). It is reported that inflamed gut releases pro-inflammatory cytokines that cross the compromised BBB to trigger low grade inflammation and inflamm-aging (Kelly et al., 2015). We therefore studied the microbiome composition of the two strains pre and post-MPTP. The outcome may be extrapolated to understand the differential prevalence of PD between the Caucasians and Asian-Indians.

\section{Materials And Methods}

\section{Experimental Animals and MPTP-HCl Administration}

We used C57BL/6J (MPTP-susceptible) and CD-1 (MPTP-resistant) mice at 15-17 week (young adults), 10-12 months (middle-aged) and 18-20 months (old/aged). All the experiments were conducted on male mice as there is a male preponderance in prevalence and incidence of PD (reviewed by Georgiev et al., 2017). They were housed under standard laboratory conditions of temperature $25^{\circ} \pm 2^{\circ} \mathrm{C}, 12 \mathrm{~h}$ light: $12 \mathrm{~h}$ dark cycle with ad libitum access to food and water. The mice received four intraperitoneal injections of MPTP-hydrochloride ( $15 \mathrm{mg} / \mathrm{kg} / \mathrm{dose})$ in saline, at 2-h intervals. The control ones received saline (Vidyadhara et al., 2017). The mice were sacrificed at days 1, 4 \& 7 after MPTP-injection and subjected to various analyses.

\section{Tissue processing for immunohistochemistry (IHC):}

Male mice anaesthetized using isoflurane were intracardially perfused with $0.9 \%$ heparinized saline followed by $4 \%$ ice-cold buffered paraformaldehyde $(0.1 \mathrm{M})$. The brains were removed and post-fixed for 48 hours at $4^{\circ} \mathrm{C}$; cryoprotected in buffered sucrose grades $(10 \%, 20 \% \& 30 \%) .40 \mu \mathrm{m}$ thick serial coronal sections of midbrains were collected on gelatin coated slides.

\section{Immunostaining:}

Two different series were used for Iba- 1 and s100- $\beta$ labeling ( $n=3-4 /$ strain/age group/experimental condition); with a section periodicity of 1 in 6 . Antigen was unmasked using sodium citrate buffer $(\mathrm{pH}-6)$ at $80^{\circ} \mathrm{C}$ for 20 min. Endogenous peroxidase was quenched using $0.1 \%$ hydrogen peroxide $\left(\mathrm{H}_{2} \mathrm{O}_{2}\right)$ in $70 \%$ methanol (20 min in dark). The non-specific binding was blocked with $3 \%$ buffered bovine serum albumin (BSA) for $4 \mathrm{hr}$ at room temperature (RT). The primary antibody exposure (0.1M PBS-TX; dilution 1:500; Table 1) lasted for 72 hours at $4^{\circ} \mathrm{C}$ in a closed chamber. Biotinylated secondary labeling ( 8 hr; dilution 1:100, Vector labs, USA; Table 1) was followed by tertiary labeling with avidin-biotin complex (Vector labs, USA; 4 hr at RT). The staining was visualized with $0.05 \%$ DAB (3'-3'-diaminobenzidine) and $0.01 \%$ nickel sulphate in $0.1 \mathrm{M}$ acetate imidazole buffer $(\mathrm{pH} 7.4)$ containing $0.01 \% \mathrm{H}_{2} \mathrm{O}_{2}$ resulting in a black colored reaction. For the second antibody, similar procedure was followed (Table 1) except that the chromation was performed exclusively with $D A B$, resulting in brown colored profiles [(TH (brown) and Iba- $1 / \mathrm{s} 100 \beta$ black)]. Negative controls were processed without adding primary antibody. The sections were mounted with DPX following alcohol-based dehydration. 
Table 1

Details of Primary and Secondary antibodies used for immunohistochemistry

\begin{tabular}{|c|c|c|c|c|}
\hline \multicolumn{2}{|c|}{ Staining modality, primary antibody and source } & \multirow{2}{*}{$\begin{array}{l}\begin{array}{l}\text { Dilution and incubation } \\
\text { time }\end{array} \\
1: 800\end{array}$} & \multirow{2}{*}{$\begin{array}{l}\text { Secondary antibody and } A B C \\
\text { kits } \\
\text { Goat Elite } A B C \text { kit, }\end{array}$} & \multirow{2}{*}{$\begin{array}{l}\text { Dilution and incubation } \\
\text { time }\end{array}$} \\
\hline Immuno-peroxidase & Anti-Goat Iba-1, & & & \\
\hline & Abcam, UK & 72 hr at $4^{\circ} \mathrm{C}$ & Vector laboratories, USA & \\
\hline & Anti-Mouse $S 100 \beta$, & $1: 800$ & $M O M * A B C$ kit, & $1: 500,3 \mathrm{hr}$ at RT \\
\hline & Sigma -Aldrich & $72 \mathrm{hr}$ at $4^{\circ} \mathrm{C}$ & Vector laboratories,USA & \\
\hline & Anti-Rabbit TH, & $1: 1000$ & Rabbit Elite ABC kit, & $1: 200,4 \mathrm{hr}$ at RT \\
\hline & Santa Cruz, USA & $72 \mathrm{hr}$ at $4^{\circ} \mathrm{C}$ & Vector laboratories,USA & \\
\hline \multirow[t]{10}{*}{ Immuno-fluorescence } & Anti-Goat Iba-1, & $1: 800$ & FITC, Sigma Aldrich,USA & $1 ; 2006-8 \mathrm{hr}$ at $4^{\circ} \mathrm{C}$ \\
\hline & Abcam UK & $72 \mathrm{hr}$ at $4^{\circ} \mathrm{C}$ & & \\
\hline & Anti-Rabbit GFAP, & $1: 500$ & Cy3, Sigma Aldrich,USA & $1 ; 2006-8 \mathrm{hr}$ at $4^{\circ} \mathrm{C}$ \\
\hline & Abcam UK & $24 \mathrm{hr}$ at $4^{\circ} \mathrm{C}$ & & \\
\hline & \multirow{2}{*}{$\begin{array}{l}\text { Anti-Rabbit Fractalkine, Abcam } \\
\text { UK }\end{array}$} & $1: 500$ & Cy3, Sigma Aldrich, USA & $1 ; 2006-8 \mathrm{hr}$ at $4^{\circ} \mathrm{C}$ \\
\hline & & 72 hr at $4^{\circ} \mathrm{C}$ & & \\
\hline & \multirow[t]{2}{*}{ Anti Mouse CNPase, Abcam UK } & $1: 1000$ & FITC, Sigma Aldrich, USA & $1 ; 2006-8 \mathrm{hr}$ at $4^{\circ} \mathrm{C}$ \\
\hline & & $72 \mathrm{hr}$ at $4^{\circ} \mathrm{C}$ & & \\
\hline & Anti Rabbit MFGE8, & $1: 800$ & Сy3, Sigma Aldrich, USA & $1 ; 2006-8 \mathrm{hr}$ at $4^{\circ} \mathrm{C}$ \\
\hline & Cloude Clone crop & 72 hr at $4^{\circ} \mathrm{C}$ & & \\
\hline
\end{tabular}

For immunofluorescence labeling, following the incubation with primary antibody, the sections were incubated in an appropriate fluorescently labeled secondary antibodies (Sigma-Aldrich, USA). The sections were then mounted using Vectashield hard set mounting medium (Vector Laboratories, USA) and images were captured using a laser scanning confocal microscope (DMIRE-TCS Leica Microsystems, Germany).

\section{Stereological quantification of cells:}

The immunoreactive (ir) cells in SNpc were quantified using optical fractionator method with an Olympus BX61 Microscope (Olympus Microscopes, Japan) equipped with Stereoinvestigator software version 8.1 (Micro-brightfield Inc., Colchester, USA). The SNpc in TH-ir midbrain sections was delineated on both the sides under 10X (Paxinos, 2013). Pilot studies determined the grid interval and counting frame size. Cells were counted in every sixth section (6-8 sections/animal) under $100 \mathrm{X}$, with a grid interval of $10000 \mu \mathrm{m}^{2}(\mathrm{x}=100 \mu \mathrm{m}, \mathrm{y}=100 \mu \mathrm{m})$ and counting frame of size $6400 \mu \mathrm{m}^{2}(\mathrm{x}=80 \mu \mathrm{m}, \mathrm{y}=80 \mu \mathrm{m})$ as per our earlier report (Vidyadhara et al., 2017). The absolute numbers were derived and coefficient of error was determined according to Gundersen et al., (1999).

\section{Immunoblotting:}

The mice ( $n=5 /$ strain/age group/experimental condition) were sacrificed by cervical dislocation and the midbrains were snap frozen in liquid nitrogen and stored at $-80^{\circ} \mathrm{C}$ till further use. The brains were thawed to $-20^{\circ} \mathrm{C}$ on a cryostat and $5 \mu \mathrm{m}$ thick sections of SN were solubilized in $100 \mathrm{ul}$ of mammalian lysis buffer $10 \%$ protease inhibitor cocktail (Sigma-Aldrich, USA). Following sonication (Q sonica, India) and centrifugation at $12000 \mathrm{~g}\left(4^{\circ} \mathrm{C}\right)$ the protein concentration in the supernatant was assayed by Bradford methodand 60ug of protein/sample was electrophoresed (Bio-Rad, USA) on a 5\%/10\% (loading/separating) denaturing gel. The proteins were transferred onto a buffered poly-vinilidine di-fluoride (PVDF) membrane (Millipore, Germany). The nonspecific staining was blocked by $5 \%$ skimmed milk protein ( 1 X TBST; $4 \mathrm{hr}$ ) followed by overnight incubation with primary antibody solution (Table 2 .). This was followed by incubation with appropriate HRP- conjugated secondary antibody for $2 \mathrm{hr}$ (Table 2.). The band was detected using chemi-luminescent substrate for HRP (Super Signal West Pico, USA) using a geldoc apparatus (Syngene International Ltd., India) and quantified using Image J 1.48 v program (Vidyadhara et al., 2017). 
Table 2

Details of primary and secondary antibodies used for immunoblotting

\begin{tabular}{|c|c|c|c|}
\hline Primary antibody & $\begin{array}{l}\text { Dilution and incubation } \\
\text { time }\end{array}$ & Secondary antibody & $\begin{array}{l}\text { Dilution and incubation } \\
\text { time }\end{array}$ \\
\hline \multirow[t]{2}{*}{ Anti iNOS, Rabbit, Abcam USA } & 1:1500, & \multirow{2}{*}{$\begin{array}{l}\text { Anti Rabbit HRP-Conjugated secondary antibody, } \\
\text { Merck }\end{array}$} & 1:2000, \\
\hline & $8 \mathrm{hr}$ at $4^{\circ} \mathrm{C}$ & & $2 \mathrm{hr}$ at RT \\
\hline \multirow{2}{*}{$\begin{array}{l}\text { Anti Fractalkine, Rabbit, Abcam } \\
\text { USA }\end{array}$} & 1:800, & \multirow{2}{*}{$\begin{array}{l}\text { Anti Rabbit HRP-Conjugated secondary antibody, } \\
\text { Merck }\end{array}$} & 1:2000, \\
\hline & $8 \mathrm{hr}$ at $4^{\circ} \mathrm{C}$ & & $2 \mathrm{hr}$ at RT \\
\hline \multirow[t]{2}{*}{ Anti $\beta$-actin, Mouse, Sigma Aldrich } & 1:2000, & \multirow{2}{*}{$\begin{array}{l}\text { Anti Mouse HRP-Conjugated secondary antibody, } \\
\text { Merck }\end{array}$} & 1:2000, \\
\hline & $8 \mathrm{hr}$ at $4^{\circ} \mathrm{C}$ & & $2 \mathrm{hr}$ at $\mathrm{RT}$ \\
\hline
\end{tabular}

\section{Enzyme-linked immunosorbent assay (ELISA):}

Protein lysates were obtained from ventral midbrains ( $n=6 /$ strain/age-group/time point/experimental condition) of post-MPTP day (d) 1 , $d 4$ and d7 mice. Both pro-inflammatory (TNF-a, IL-6, IL-1 $\beta$ ) and anti-inflammatory cytokines (TGF- $\beta$, IL-4, 1L-10) were evaluated as per the manufacturer's instructions (Ray Biotech, Inc, USA). MAO-A and MAO-B kits were obtained from Cloud- clone Corp, (Wuhan, China). Briefly 100 $\mu$ l of standards/samples were added to each well except blanks/standards $\left(2.5 \mathrm{hr}, 37^{\circ} \mathrm{C}\right)$. Thereafter, $100 \mu \mathrm{l}$ of biotinylated secondary antibody was added $(1 \mathrm{hr}, \mathrm{RT})$ with gentle shaking followed by streptavidin $(100 \mu \mathrm{l})$. The chromation was achieved with TMB (3,3',5,5'-tetramethylbenzidine) one-step substrate reagent. The reaction was stopped with $50 \mu$ l stop solution/well and the absorbance was measured immediately at $450 \mathrm{~nm}$ (TECAN, Austria). All standards and samples were run in duplicates.

\section{Electron microscopy}

The mice ( $n=3$ /strain/age group/experimental condition) were anesthetized with isoflurane and transcardially perfused with a buffered mixture of $2.5 \%$ glutaraldehyde and $2 \%$ PFA (in $0.1 \mathrm{M}$ phosphate buffer, $\mathrm{pH} 7.4$ ). The dissected substantia nigra was cut into smaller pieces, post-fixed with $1 \%$ osmium tetroxide at RT and dehydrated in grades of ethyl alcohol followed by clearing in propylene oxide. Infiltration (1:1 mixture of araldite and propylene oxide, overnight at RT, on a rotator) was followed by exposure to pure araldite for $4.5 \mathrm{~h}$. The resin embedded tissues (flat embedding molds) were allowed to polymerize $\left(60^{\circ} \mathrm{C}, 48 \mathrm{hr}\right) .1 \mu \mathrm{m}$ semi thin sections, were stained with $1 \%$ toluidine blue to verify the region of interest and $60 \mathrm{~nm}$ ultrathin sections (Leica, Ultramicrotome, Austria) collected on copper grids were stained using saturated uranyl acetate for $1 \mathrm{hr}$ and $0.2 \%$ lead citrate (5-7 min). The washed and air dried sections were examined under Transmission Electron Microscope (FEI, TECNAI G2 Spirit BioTWIN, Netherlands) and images were captured using Mega View-III CCD camera (Shruthi et al., 2017).

\section{Mouse fecal microbiome analysis}

The composition of gut microbiota was analyzed for fecal microbiome; pre- and post-MPTP. Mice $(n=3 /$ strain; total $n=12)$ were housed separately in autoclaved cages (without bedding) and allowed to defecate normally. The first three fecal pellets were collected into sterile cryovials. The samples were snap frozen in liquid nitrogen and stored at $-80^{\circ} \mathrm{C}$. $16 \mathrm{~s}$ rRNA metagenome sequencing was performed after a quality check (Clevergene Biocorp Pvt. Ltd. Bangalore, India).

\section{Statistics:}

The ELISA related data was analyzed using Repeated Measures ANOVA followed by Mann-Whitney U test. The rest was analyzed using two- way ANOVA followed by Tukey's post hoc test using SSPS software and GraphPad Prism software (GraphPad Software version 6.01 Inc, USA). A p-value < 0.05 was considered as statistically significant. The data was expressed as mean \pm standard error of mean (mean \pm SEM).

\section{Results}

\section{Differences in number of glia:}

\section{Microglia}

The strain-specific differences in the neuronal numbers of substantia nigra noted earlier (Vidyadhara et al., 2017) prompted us to verify if the differences extended to baseline numbers of glia. Iba-1 stained the microglia well (Fig. 1A\&B; arrows), including their processes and were easily discriminated from neurons (arrowheads) in the vicinity. Based on unbiased stereology, CD-1 nigra had relatively fewer Iba- 1 ir cells across aging although not statistically significant (Fig. 1C; $18.2 \%$ at young age; $14 \%$ at middle-age and $8.5 \%$ at old age). In response to MPTP, an increase in microglia was noted in old animals of both strains [C57BL/6J MPTP (young vs. middle-aged \#\# $p<0.01$, young vs. old \# $p<0.05$ ), CD-1 MPTP (young vs. old $\$ p<0.05$ )].

\section{Astrocytes}

The astrocytes appeared much darkly stained (Fig. 1D\&E; arrows) compared to the TH ir dopaminergic neurons (arrowheads). The control CD-1 mice substantia nigra had more s100ß positive astrocytes (Fig. 1B) than the young (Fig. 1C\&D, 14.68\%) and old C57BL/6J (Fig. 1C\&D, 10.67\%). Under normal conditions, in C57BL/6J, the numbers increased significantly from youth to middle-age (C57BL/6J young vs. middle-aged \#\# $p<0.01 ; 24.06 \%$ ) to stabilize later (C57BL/6J young vs. old \#\# $\mathrm{p}<0.01 ; 26.75 \%$ ). In contrast, the CD-1 substantia nigra showed a gradual age-related increase in the astrocytes (CD-1, young v/s middle age, $11.64 \%$; young vs. old $\$ \$ p<0.01 ; 22.32 \%$ ). MPTP caused a significant increase in the numbers at all ages, in both the strains; notably Loading [MathJax]/jax/output/CommonHTML/fonts/TeX/fontdata.js :ontrol vs. MPTP, 15.8\%). 
II. Cytokine expression in the ventral midbrain: Increase in the numbers of microglia and astrocytes with age and in response to MPTP led us to hypothesize that gliosis may induce neuroinflammation. We therefore estimated the levels of pro and anti-inflammatory cytokines.

\section{Pro-inflammatory cytokine levels are relatively higher in C57BL/6J}

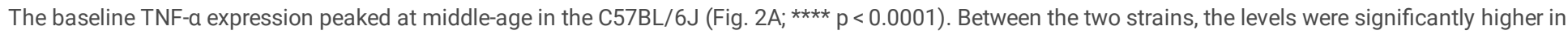
the old C57BL/6J (C57BL/6J vs.CD-1 * $<<0.05)$. MPTP caused a sizeable upregulation in the C57BL/6J substantia nigra across ages and at all the time

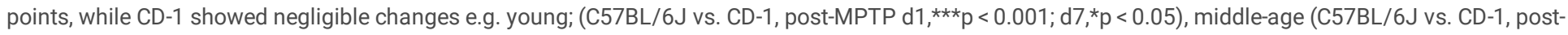

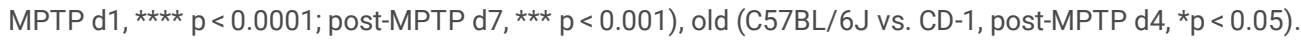

Both the strains showed comparable patterns of IL-6 expression with age and following MPTP (Fig. 2B), with a significantly higher expression at middle-age (C57BL/6J young vs. middle-age ${ }^{\# \# \# p<0.0001) ; C D-1 ~ y o u n g ~ v s . ~ m i d d l e-a g e d ~}$

$\mathrm{p}<0.0001$ ) and down-regulation at old-age [C57BL/6J (middle-aged vs. old ${ }^{\# \# \#} \mathrm{p}<0.001$ ); CD-1 (middle-aged vs. old aged

$p<0.0001)]$. MPTP elicited similar responses in both strains at young as well as at middle-age when an upregulation was appreciated at 4 days post-MPTP $(p$

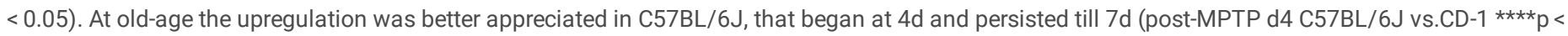

0.0001 , post-MPTP d7 C57BL/6J vs.CD-1 *p<0.05).

IL-1 $\beta$ showed a significant reduction with aging in both strains (Fig. 2C; C57BL/6J young vs. middle-aged \#\#\# $\mathrm{p}<0.0001 ; \mathrm{CD}-1$ young vs. middle-aged

$\mathrm{p}<0.0001 ; \mathrm{C} 57 \mathrm{BL} / 6 \mathrm{~J}$ middle-aged vs. old $\# \# \#$ p $<0.001$; CD-1 middle-aged vs. old

$\mathrm{p}<0.001$ ). MPTP-injected young C57BL/6J showed an acute increase at d1 (control vs. post-MPTP day1 \# $<0.05$ ) followed by a reduction till d7 (post-MPTP

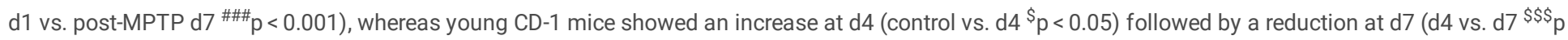
$<0.001$ ). At middle-age there were no perceptible differences between the strains as both showed a significant reduction at days 1-4 post MPTP followed by a

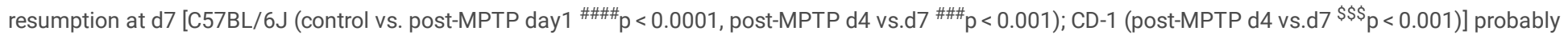

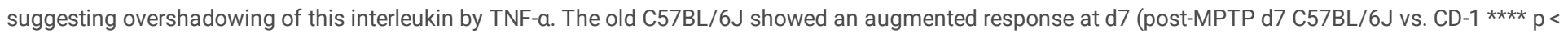
0.0001) probably suggesting a late response by activated astrocytes.

\section{B. Anti-inflammatory cytokine levels are relatively lower in C57BL/6J:}

The basal level expression of anti-inflammatory TGF- $\beta$ was significantly lower in C57BL/6J at all age points [(Fig. 3A C57BL/6J vs. CD-1; young *p < 0.05), middle-aged $(* \star \star * p<0.0001)$, old $(* \star \star \star p<0.0001)$ ]. In response to MPTP, CD-1 showed a profound increase in expression in the young [post-MPTP $d 7$

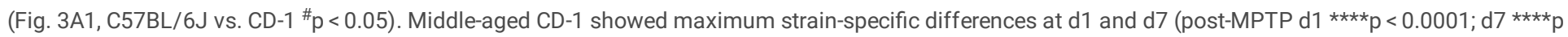

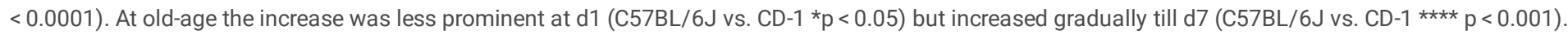

Both the strains showed a significant increase in IL-4 expression at middle-age, with relatively higher levels in C57BL/6J (Fig. 3B; C57BL/6J vs. CD-1 * $<$ < 0.05). In the young CD-1, the expression decreased at d1 post-MPTP, yet causing a significant upsurge at d4. C57BL/6J showed comparatively higher IL-4 expression at old age in response to MPTP at d4 (C57BL/6J vs.CD-1 ** $p<0.01)$ and d7 (C57BL/6J vs.CD-1 * $p<0.05)$.

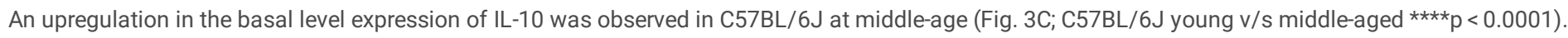
MPTP caused a significant upsurge in IL-10 expression in C57BL/6J at middle and old age (middle-aged post-MPTP d1\&4 **p<0.01; d7 *p< 0.05). At old age, MPTP elicited an increase only at $d 4$ (C57BL/6J vs. CD-1 *** $\mathrm{p}<0.001)$, and d7 (C57BL/6J vs. CD-1 *** $\mathrm{p}<0.0001)$.

\section{III: Differences in enzyme expression:}

\section{A: MAO-A and MAO-B levels:}

MAO-A positive punctae were localized to the cytoplasm (Fig. 4A1, red) of the DA neurons (Fig. 4A2) as also in the neuropil (Fig. 4A3 pink coloration in neurons and red in the neuropil). The basal levels were higher in the substantia nigra of young CD-1 (C57BL/6J vs. CD- 1 *p $<0.05)$, however middle-age saw a

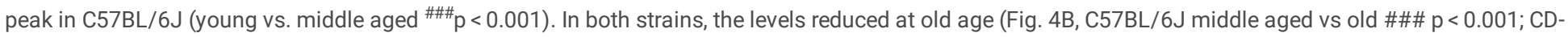
1 middle aged vs. old $\$ \$ p>0.01)$. MPTP induced a notable increase in the young C57BL/6J; although both strains showed moderate enhancement at all ages (C57BL/6J control vs. MPTP \#\#\# $<$ 0.001).

The GFAP expressing glia (Fig. 4B1, green) showed MAO-B immunoreactivity (Fig. 4B2, red Fig. 4B3 merge, yellowish green). MAO-B levels were also higher in young CD-1 than C57BL/6J (Fig. 4 fD). An age-associated gradual down-regulation in CD-1 was contrasted by a gradual increase in C57BL/6J, although statistically significant.In summary, MPTP administration up regulated both MAO-A and MAO-B level in both strains

\section{B: Inducible nitric oxide synthase (iNOS):}

Iba-1 immunopositive microglia (Fig. 5E1; green) expressed iNOS (Fig. 5E2; red) in addition to some non-microglial cells (Fig. 5E3 merge; red). The antibody showed a single band of $140 \mathrm{KDa}$ (Fig. 5I). Young CD-1 had significantly higher basal iNOS (Fig. 5F; C57BL/6J vs. CD- 1 *p < 0.05). At middle-age both strains showed a moderate increase in expression. With aging, the CD-1 mice showed mild decrease in iNOS expression whereas C57BL/6J maintained the levels attained at middle age. MPTP caused iNOS augmentation across ages in both strains (middle-aged C57BL/6J control vs. MPTP \# $\mathrm{p}<0.05$, old C57BL/6J control vs. MPTP \# $\mathrm{p}<0.05$, old CD-1 control vs. MPTP $\$ p<0.05)$.

Loading [MathJax]/jax/output/CommonHTML/fonts/TeX/fontdata.js 


\section{C: Fractalkine:}

Fractalkine was localized to the DA neuronal cytoplasm (Fig. 5G1-G3). The antibody showed a band at 140 KDa (Fig. 5J). The CD-1 substantia nigra had moderately high levels at all the ages, compared to $\mathrm{C} 57 \mathrm{BL} / 6 \mathrm{~J}$. A decrease was evident in both strains after middle age (Fig. 5H, $\mathrm{C} 57 \mathrm{BL} / 6 \mathrm{~J}$ middle-age vs. old $\# p<0.05, C D-1$ middle-age vs. old $\$ \$ p<0.01)$. MPTP elicited an increase in both the strains, but more appreciably in the young (C57BL/6J, young adults vs. middle-aged \#\#\# $\mathrm{p}<0.0001$, middle-aged vs. old \#\#\# $\mathrm{p}<0.0001)$.

\section{D: Hemeoxygenase-1 (HO-1):}

The young CD-1 midbrains showed mildly higher levels of HO-1 which reduced following MPTP. Both strains showed a significant up-regulation at middle-age that persisted till old age (Fig. 5k, C57BL/6J young vs. middle-aged \#\#\#\# $p<0.001, \mathrm{CD}-1$ young vs. middle-aged $\$ \$ \$ p<0.001)$. Aged mice of both strains showed an increase in H0-1 expression in response to MPTP; moderate in C57BL/6J; significant in CD-1 (CD-1 control vs. MPTP\$\$ p < 0.001).

\section{IV: Age-related and MPTP-induced ultrastructural changes:}

\section{A: Effects on Substantia Nigra Neurons}

The mitochondria of the young and middle aged C57BL/6J were relatively larger than those of the elderly (Fig. 5A,E\&I). MPTP induced mitochondrial shrinkage at all ages. (Fig. 5 compare A\&B and E\&F, I\&J; 'M' arrows). However in CD-1 they were well preserved with age (Fig. 5 compare C\&D and G\&H, K\&L; ' $\mathrm{M}$ ' arrows) and even longer in response to MPTP (Fig. $5 \mathrm{H}, \mathrm{H} 1$ and $\mathrm{L}$, arrow). The endoplasmic reticular (ER) strands shortened with age and in response to MPTP in C57BL/6J (Fig. 5, compare A\&B and E\&F, I\&J; ER).Whilst MPTP induced ER dilatation in the young (Fig. 5; D2) and middle-aged CD-1 (Fig. 5H; ER). The normal aged CD-1 showed presence of ER arrays (Fig. 5K1). Interestingly, the aged mice of both strains showed presence of several Golgi apparatus units (Fig. 5I 'Go'). In the MPTP-administered aged C57BL/6J they appeared circular and possessed bloated saccules with "pearl necklace like appearance" (Fig. 5, F\&H; J\&L1; 'Go'). In the older C57BL/6J apoptotic bodies were noted (Fig. 5, I1; 'Ab') while in CD-1, the neuronal nucleus was often crenellated. The neurons of MPTP-injected young CD-1 harbored several lysosomes, which were conspicuously absent in the neurons of young C57BL/6J (Fig. 5B\&D 'Ly').

\section{B: Astrocytes}

The astrocytic nuclei were larger but not uniformly ovoid/round like those of oligodendrocytes. Their nuclear chromatin was fine and granular. The cytoplasm was sparse and granular within the perinuclear zone (Luse SA, 1956), but more electron dense than the neuronal cytoplasm. The nucleus remained euchromatic through aging and in response to MPTP. The astrocytes of young and middle aged C57BL/6J had numerous long and tubular cytoplasmic mitochondria (Fig. 6, 'M', compare A\&B and E\&F) whereas those in the myelinated axons were spherical ('Ma'). In CD-1, the cytoplasmic mitochondria were oval (Fig. 6, compare C\&D and G\&H). The astrocytic mitochondria were relatively longer in the old CD-1 (Fig. 6, compare E\&G). The ER was relatively well maintained through aging and in response to MPTP in both strains. Golgi saccules were semicircular and dilated in the middle aged MPTP-injected CD-1 (Fig. 6, Go, compare G\&H and K\&L). The scale bar is $1 \mu \mathrm{m}$ for all micrographs except ' $\mathrm{K}$ '.

\section{C: Microglia}

Most microglia were present near the blood vessels and had electron dense cytoplasm with a bean shaped nucleus. Heterochromatin nets and electron dense pockets were noted along the nuclear perimeter. In the young, the nuclei were euchromatic (Fig. 7, A-D; 'M-nu') while those of middle aged and old, were electron dense (Fig. 7, E-L; 'M-nu'). In the cytoplasm and neighboring tracks of MPTP-injected young C57BL/6J, long tubular mitochondria were noted (Fig. 7B, 'M'). The young CD-1 showed phagocytotic microglia along with the engulfed cells (Fig. 7D; 'EC') as also the MPTP-injected old C57BL/6J (Fig. 7J). Interestingly amoeboid "dark cells" were seen around blood vessels in both strains after middle age (Fig. 7E, G and I; 'DC') and had thin rim of cytoplasm containing few organelles.

\section{Thickening of the vascular basement membrane:}

Blood vessels showed comparable membrane architecture in the young mice of both strains in control conditions as well as upon MPTP challenge (Fig. 8A-D). At middle age, MPTP injected C57BL/6J showed a membrane discontinuity suggesting a possible breach (E vs $F$, arrowheads) and thickening of basement membrane in C57BL/6J (arrows, E vs F) but not in CD-1 (F vs H). At old age, MPTP caused thickening of vascular basement membranes in both strains (arrows, I vs J; K vs L).Macrophage-like-cells (** I\&J) were seen in the lumen in old C57BL/6J. Dark cells (DC) were noted too.

\section{MPTP caused microbial dysbiosis}

It has been observed that inflamed gut releases pro-inflammatory cytokines which can cross the compromised BBB and thus trigger low grade inflammation and inflamm-aging (Kelly et al., 2015). We therefore studied the microbiome composition of the two strains pre and post-MPTP. The metagenome data analysis showed differences in the relative abundance of fecal microbiota between C57BL/6J and CD-1 mice (Fig. 9). The heat map showed that the genus/species belonging to Streptococcus, Lachnospiraceae, Bacteriodes, Candidatus, Ruminococcaceae were more abundant in the C57BL/6J whereas in CD-1 those belonging to Bacteriodales, Lactobacillus and Prevotellaceae were more and those of Prevotellawere less. MPTP injection increased the Prevotellaceae and Lachnospiraceaespp in CD-1 and Prevotella, Lachnospiraceae, Bacterodales, Candidatus-Saccharomonasand Ruminococcus population in C57BL/6J.

\section{Discussion}




\section{The baseline number of microglia and astrocytes vary between strains:}

Microglia are selectively more populous in the hippocampus and SNpc (Lawson et al., 1990), the target regions of the common age-associated diseases like Alzheimer's and Parkinson's disease respectively; endorsing their role in neurodegeneration and susceptibility. In aging mice, microglial priming is equated to an immune vigilant state that results in their de-ramification, hypertrophy, increase in numbers and exaggerated response to sub-threshold challenges. They synthesize ROS and pro-inflammatory cytokines like TNF-a, IL-6, IL-1 $\beta$; while curtailing the synthesis of anti-inflammatory cytokines (Damani et al., 2011; Grabert et al., 2016). The presence of more microglia at middle age corroborate with the possibility of a pre-inflammed status and presence of damage associated molecular patterns (DAMPs) in-situ, thereby inflammasomes too may be vital in determining susceptibility. We found that normal C57BL/6J mice have fewer substantia nigra neurons (Vidyadhara et al., 2017) and more baseline apoptosis (Yarreiphang et al., 2020 unpublished data) vis-à-vis the CD-1 mice. Neuronal apoptosis flags the entry of microglial precursor cells into the zebra fish brain (Casano et al., 2016) hence, the higher number of microglia in C57BL/6J substantia nigra complement higher apoptosis and suggest higher baseline susceptibility.

Aging selectively affects the already sparse astrocytes in the substantia nigra, as against the other mesencephalic niche (Damier et al., 1996). Under physiological conditions, they secrete trophic factor GDNF (Grondin et al., 2003), therefore the presence of fewer astrocytes in young C57BL/6J and an acute increase in their numbers at middle-age that persisted till old age, suggest reduced neuroprotection and gliosis-assisted inflamm-aging linked with cytokines. Whereas, the sedate increase in astrocytic numbers in CD-1 implicate moderate age-related changes in the milieu. Excessive cytokine releasing glia recruit their neighbors, to set off a vicious cycle, wherein the pro-inflammatory environment becomes self-propagating (Noh et al., 2014).

\section{Pro-inflammatory cytokine levels are higher in the susceptible strain:}

DA neurons are sensitive to the pro-inflammatory cytokine TNF-a (Sriram et al., 2006); which is primarily secreted by microglia and in synchrony with IFN-Y/IL$1 \beta$ induces neurodegeneration (Chao et al., 1995; Jeohn et al., 1998). Thereby, the dying neurons hold microglia in their cytotoxic state, to escalate synthesis of TNF-a leading to a self-perpetuating neuroinflammation. The supernumerary microglia and the persistently higher baseline TNF-a level through aging in C57BL/6J, may be interlinked and their microglia may be consistently primed, leading to increased neuronal susceptibility. Elevated TNF-a levels are reported in mouse models as well as in autopsied brain tissue/CSF of PD patients (Mogi et al., 1994). Low levels of TNF-a are protective (Chertoff et al., 2011). Thus, low levels in CD-1 in addition to being a mark of low susceptibility, may be potentially neuroprotective. Our auxiliary finding of up-regulation of TNF-a at middle-age in C57BL/6J, indicates that imbalance in cytokine milieu precedes senescence.

IL-6, a pleiotropic cytokine, is up-regulated in SN, CSF and serum of PD patients (Blum-Degena et al., 1995; Hofmann et al., 2009). Conversely, Bolin et al., (2002) reported amplified MPTP-susceptibility in IL-6 $6^{-/-}$mice. In our study, since IL-6 followed a similar age-related expression pattern in both strains, it may not dictate baseline susceptibility. Yet, the differences following MPTP validate its role as a toxicity signal. The middle-age demarcates a period of enhanced susceptibility. MPTP-induced IL-6 expression in old C57BL/6J at later stages of the challenge, suggests a link with astroglial responses or excessive neuronal death.

Reactive microglia in the degenerating SN overproduce IL-1 $\beta$, to activate astrocytes and promote iNOS secretion (Chhor et al., 2013). Chronic IL-1 $\beta$ expression in Wistar-rat substantia nigra induced progressive neurodegeneration, microgliosis and motor disabilities(Ferrari et al., 2006). The acute increase in IL-1 $\beta$ at $\mathrm{d} 1$ post-MPTP that persists till d7 in the young C57BL/6J vis-à-vis CD-1endorses immediate microglial priming in the former. Thus, the mice differ in the immediacy or respondence indices. Moreover noticeably higher basal TNF- $\alpha$ in C57BL/6J at middle and old age underlines its role in basal susceptibility, aging and neurodegeneration, whereas, IL-6 and IL-1 $\beta$ appear to be responders to MPTP and hence involved in pathogenesis.

\section{The resistant strain has higher levels of anti-inflammatory cytokines:}

TGF $\beta 1$ inhibits microglial activation and protects DA neurons against MPTP-toxicity (Arimoto et al., 2007; Pintado et al., 2011). The low baseline TGF- $\beta$ expression in C57BL/6J through aging and following MPTP, suggest that the sensitive strain is ill equipped against neuroinflammation. MPTP-elicited upregulation of TGF- $\beta$ in middle-aged CD-1 at days 1 and 7 suggest a microglia mediated initiation that persists till the late activation stage of astrocytes. Since CD-1 have more substantia nigral DA neurons and a sizeable number resists MPTP (Vidyadhara et al., 2017), higher TGF 1 levels allude to neuroprotection and DA neuronal survival.

IL-4 protects DA neurons against MPP ${ }^{+}$toxicity by up-regulating CD200, a microglial resting signal (Lyons et al., 2009). The significant upsurge at d4 in MPTPinjected young CD-1, suggests an auxiliary course by the microglia. Interestingly, the elevated response at late stages of MPTP exposure in old C57BL/6J, may be a delayed attempt at neuroprotection (reviewed by Hirsch and Hunot, 2009). In view of the pronounced increase in MPTP-sensitive C57BL/6J at middle-age, it is likely that IL-4 may also have a pro-inflammatory role. Bok et al., (2018) while showing the microglia-specific expression of IL-4 demonstrated an LPSinduced upregulation in expression and rescue of substantia nigra DA neuronal loss by antibodies against IL-4.

IL-10 stimulates CD200 expression in neurons and induces astrocytes to synthesize anti-inflammatory TGF- $\beta$. It inhibits microglial synthesis of TNF-a, NO and ROS, in-vitro, to neutralize oxidative stress (Balasingam and Yong, 1996; Ledeboer et al., 2002). The up-regulated baseline IL-10 expression in C57BL/6J and upon MPTP at middle/old-age may be a compensatory increase. However, these increases do not parallel a raise in TGF- $\beta$ levels; hinting at a failed rescue attempt. Thus, the acute increase in basal levels of both TNF- $\alpha$ and IL- 6 as well as the anti-inflammatory IL-1 $\beta$ and IL-10 expound a hovering imbalance of proand anti-inflammatory cytokines, at middle age; therefore tempting one to speculate that middle-age imitates the prodromal period in the susceptible strain.

\section{Strain specific variability in enzyme responses:}

MAO-B inhibitors are promising candidates in the treatment of early-PD(Rabey et al., 2000). MAO-B level increases with age (Irwin et al., 1997)and is doubled in SN of PD patients (Damier et al., 1996). The age-associated up-regulation in MAO-A and MAO-B levels in C57BL/6J suggests a functional decline. The Loading [MathJax]/jax/output/CommonHTML/fonts/TeX/fontdata.js lenomenon that assists in combating MPTP-related stress. Although the reasons for higher basal 
level of MAO-A and MAO-B in young CD-1 are unclear, it may be due to higher number of neurons or DA terminals and astrocytes in CD-1striatum or it may be a bystander susceptibility marker. MPTP may cause a feed-forward effect on glia, setting off a toxicity cycle.

HO-1 a cytoprotective, anti-apoptotic, and anti-inflammatory enzyme; down-regulates pro-inflammatory cytokines like TNF-a1 and IL-1 $\beta$ and up-regulates antiinflammatory cytokine IL-10 in-vitro (Doré et al., 1999; Petrache et al., 2000). Predominantly expressed by astrocytes (Dwyer et al., 1995), it is positively correlated with aging and PD (Schipper et al., 1998). It was projected as a potential biomarker due to high levels in the patient saliva(Song et al., 2018). HO-1 overexpression tendered neuroprotection in $\mathrm{MPP}^{+}$treated Parkinsonian rats via BDNF and GDNF(Hung et al., 2008). Thus, the gradual age-related increase in its levels in C57BL/6J and in response to MPTP may indicate a cellular offset response to oxidative stress. Induction of iNOS causes NO release, which when protracted triggers oxidative damage in DA neurons (Nathan and Xie, 1994). The MPTP-induced up-scaling of iNOS in both strains supports this hypothesis.

The higher baseline iNOS and MAO-B levels alongside lower HO-1 levels in CD-1 are presently un-explained; yet it is likely that higher number of astrocytes and neurons could be the reason. Alternatively, these may be markers of sub-threshold susceptibility.

Neurons secrete the chemokine fractalkine (CX3CL1), to maintain microglia in resting state (Cardona et al., 2006). The inherently higher fractalkine levels in CD-1 indicate their healthy status. Age-related decrease in C57BL/6J relays enhanced phagocytic signals, an indirect indicator of neuronal loss.

Overexpression in response to MPTP in young mice implies the activation of compensatory responses during youth which decline with age.

\section{Surviving neurons harbor strain-specific ultrastructural signatures:}

This is the first study on the alterations in the ultrastructure of mice substantia nigra with aging and in response to MPTP. The age-related and MPTP-induced shrinkage of mitochondria in C57BL/6J validates the ensuing mitochondrial dysfunction and aging as a risk factor for PD. Upregulation of mitochondrial fission protein dynamin-like protein 1 (DLP1/DRP1) as well as downregulation of fusion proteins Mfn1 and Mfn2 were noted in the substantia nigra of PD patients (Zhao et al., 2017). We earlier found higher DRP-1 levels in the lateral/ventral substantia nigra of C57BL/6J, earmarking the inherent susceptibility of the mitochondria. The well-preserved mitochondrial structure and size with age and upon-MPTP in CD-1 complements the higher HSD-10 (mitochondrial fusion-associated protein) expression (Seshadri and Alladi, 2019). Elongated mitochondria are deft in energy generation (reviewed by Galloway et al., 2010) and calcium uptake (Lewis et al., 2018). In Caenorhabditis elegans, modulation of mitochondrial proteases SPG-7 and PPGN-1 enhanced mitofusion (Chaudhari and Kipreos, 2017) to extend their overall survival. Thus enhanced HSD-10 expression (Seshadri and Alladi, 2019)and elongated mitochondria in CD-1 neurons may be the survival modalities.

Both fragmented and dilated ER are major pathological notations in A53TaS Tg mice substantia nigra (Colla et al., 2012) and rotenone model of subcutaneous administration (Zhang et al., 2017), suggesting ER dysfunction in PD. ER arrays in old CD-1 could be rejoinders of enhanced protein synthesis to compensate for the concurrent protein loss or mis-folding. The MPTP-induced ER shortening in C57BL/6J and its dilation in CD-1 suggests existence of strainspecific differences as well as different aspects of ER dysfunction in PD, which needs to be studied in detail.

The presence of many intact Golgi units in the aged substantia nigra of both strains suggest a compensatory increase to circumvent age effects on protein packaging and post-translational processing. The "pearl necklace like globose" bloated saccules of Golgi units upon MPTP-injection in old C57BL/6J, hint at disease-induced functional impairment. Knockdown of adhesion proteins like GRASP 55/65 cause Golgi cisternae swelling(Lee et al., 2014). Simulation studies suggest that aberrations in biophysical properties like osmotic pressure, adsorption and adhesion energies, and precise vesicle addition frequency in addition to biological properties like rim stabilizer proteins cause abnormal self-organization into circular/fused Golgi complexes (Tachikawa and Mochizuki, 2017). The presence of lysosomes in neurons of MPTP-injected young CD-1 that were visibly fewer in C57BL/6J may suggest either the activation of lysosomal/UPR pathway or lysosomal accumulation due to impaired late endocytic pathway (Guerra et al., 2019). The apoptotic bodies in older C57BL/6J, signal the occurrence of age-associated apoptosis. The crenellated nuclei in MPTP-injected old CD-1, suggest necroptosis as seen in striatal cells of a Huntington's mouse (Turmaine et al., 2000). Thus, majority of organelles were affected in C57BL/6J both with aging and upon MPTP indicating affliction of many cellular processes. Contrarily, elongation of mitochondria, preservation of Golgi apparatus and presence of lysosomes may be pointers of resilience in CD-1. Amongst the organellar defects, ER dilation is a sure sign of susceptibility in CD-1.

\section{Elongation of glial mitochondria, a distinctive feature of pathogenesis:}

In an interesting spin off, unlike the neurons, ultrastructure of glial organelles was preserved during aging and following MPTP. The glial mitochondria that were smaller and fewer in controls C57BL/6J, appeared enlarged/elongated in response to MPTP. Hoekstra et al., (2015) showed a reduction in fission protein DLP1/DRP1 in both neurons and astrocytes in PD cortex along with fused elongated mitochondria in primary cortical astrocytes transfected with DLP1-siRNA. Co-culturing them with cortical neurons caused neuronal atrophy and excessive calcium release; suggesting neurotoxic effect of fused astrocytic mitochondria. In a stroke model, the penumbral astrocytes displayed hypertrophic and polarized processes; elongated mitochondria and lost their neuroprotective ability (Fiebig et al., 2019). Overexpression of mutant ubiquitin (UBB +1$)$ protected astrocytes from oxidative stress and $\mathrm{H}_{2} \mathrm{O}_{2}$-induced cell death by destabilizing mitochondrial fission-specific proteins, leading to mitochondrial fusion (Yim et al., 2014). Although the exact corollaries are not clear, the combination of astrogliosis and increased pro-inflammatory cytokines; tempts one to speculate that elongated mitochondria assist astroglial propagation into neurodegenerative sequels.

Mitochondrial elongation in microglia of MPTP-injected young C57BL/6J mice, may have similar neurodegenerative consequences. LPS-activated mouse cerebral microglia, stimulate DRP-1 and ROS synthesis in-vitro, to elongate tract borne mitochondria (Katoh et al., 2017). The dilated Golgi apparatus in microglia indicate that while aggravating neuroinflammation, the microglial protein packaging process is also affected. Under chronic stress, in aging and in AD; microglia had condensed, electron dense cytoplasm and nucleoplasm which imparted a striking "dark" appearance (Bisht et al., 2016). Dark cells noted in both strains from middle age, may have similar pathological objective. In most cells, the cytoplasm appeared as a thin rim, reducing the scope to visualize

Loading [MathJax]/jax/output/CommonHTML/fonts/TeX/fontdata.js 
Our findings of mitochondrial elongation may have clinical implications. For instance, the antioxidant coenzyme Q10 (CoQ10) supports mitochondrial function while reducing the DA neuron loss in an animal model of PD (Spindler et al., 2009), yet it failed in the phase III clinical trials (The Parkinson Study Group QE3 Investigators 2014). Similarly, CoQ10 derivative MitoQ also failed (Snow et al., 2010). It is likely that these molecules stabilized the glial mitochondria too, thereby nullifying the neuronal outcome. It is vital to study the glial mitochondrial responses in isolation and on a temporal scale, to better understand the phenomenon.

\section{Earlier age at onset of MPTP-induced basement membrane features in C57BL/6J:}

MPTP caused basement membrane thickening similar to that reported in PD (Farkas et al., 2000). Presence of gaps in the middle aged C57BL/6J and old CD1 implies that the blood-brain barrier (BBB) is vulnerable earlier in life in C57BL/6J, an additional signal of negative impact of aging or on the gut microbiota (Montagne et al., 2015) or of inherent susceptibility.

\section{Gut microbiome composition is distinct in the two strains:}

The gut microbiome modulates the formation of BBB, neurogenesis, microglial maturation and also influences brain homeostasis and behavior (Heijtz et al., 2011; Erny et al., 2015). Inflamed gut releases pro-inflammatory cytokines that cross the compromised BBB to trigger low grade inflammation and inflammaging(Kelly et al., 2015). PD patients show a reduction in microbes of Prevotellaceae, Lachnospiraceae and Ruminococceae family etc. alongside an increase in Enterobacteriaceae, Bifidobacteriumetc. The abundance of Prevotella, Lachnospiraceae, Enterobacteriaceae and Bacterodales population in C57BL/6J visà-vis Prevotellaceae in CD-1 mice upon MPTP-injection was a serendipitous outcome that validates strain-typical differences in fecal microbiome responses while post-MPTP increase in Prevotellaceae portrays defense mechanisms in the latter. The abundance of Enterobacteriaceae correlated positively with the severity of postural instability and gait difficulty in PD patients (Scheperjans et al., 2015). Post-MPTP worsening of motor deficits is more prominent in C57BL/6J than CD-1 (Vidyadhara et al, 2019). Thus, fecal microbiome is a reliable non-invasive marker of susceptibility.

\section{Conclusion}

In summary, neuro-glial interactions, senescence related changes in glia, cytokine levels etc. are vital determinants of neuronal survival and differ greatly in the MPTP-resistant C57BL/6J and MPTP-susceptible CD-1 white mice strain. The intended use of male animals for the study may be a limiting factor, however in view of the male preponderance of the disease; our observations provide vital clues of disease pathogenesis. Besides, female mice are also known to show higher fatality in response to MPTP, due to differences in peripheral metabolism of MPTP; independent of its effects on dopaminergic neurons. It is also pertinent to compare these factors between male and female animals to understand the premise for neuroprotection in females.

Our findings in general, may be extrapolated to different human populations that are either vulnerable or resistant to PD. For instance, in our study, the CD-1 represents Asian-Indians who have inherently lower prevalence rate than the Caucasians. The prominent differences in pro- and anti-inflammatory cytokine levels at middle-age suggests that by design, the middle-age milieu is acquiescent to neurodegeneration and may well be the critical soft period for the onset of neurodegenerative diseases. It is likely that senescence may result from differences in the in-situ inflammasomes, which merit detailed investigations. The pathogenesis is effectively assisted by the diabolic differences between the neuronal and glial mitochondria. The differences in fecal microbiome highlight the possibility of using it as a non-invasive marker of susceptibility. Thus glia are major players in aging and disease and may explain the ethnic bias in prevalence of PD.

\section{Abbreviations}

MPTP: 1-methyl-4-phenyl-1, 2, 3, 6-tetrahydropyridine

BBB: Blood brain barrier

BSA: Bovine serum albumin

Coenzyme Q10: CoQ10

D1: day 1 post-MPTP:

D4: day 4 post-MPTP

D7: day 7 post-MPTP

DAMP Damage associated molecular patterns

DLP1/DRP1: Dynamin-like protein 1

ELISA :Enzyme-linked immunosorbent assay

Endoplasmic reticular ER

GFAP: Glial Fibrillary Acidic Protein

$\mathrm{H}_{2} \mathrm{O}_{2}$ : Hydrogen peroxide

Loading [MathJax]/jax/output/CommonHTML/fonts/TeX/fontdata.js 
HO-1:Hemeoxygenase-1

Iba-1: Ionized calcium-binding adaptor protein-1

IFN-ץ: Interferon- $\gamma$

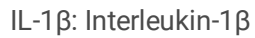

iNOS: Inducible nitric oxide synthase

Ir: immunoreactive

MAO-A : Monoamine oxidase A

MAO-B : Monoamine oxidase B

$\mathrm{MPP}^{+}: 1$-methyl-4-phenylpyridinium

PD: Parkinson's disease

PVDF: Poly-vinilidine di-fluoride

$\mathrm{RT}:$.Room temperature

SNpc: Substantia nigra pars compacta

TMB: 3,3',5,5'-tetramethylbenzidine

TNF-a: Tumor necrosis factor

\section{Declarations}

Ethics approval: All the experimental protocols on mice were approved by the Institutional Biosafety committee and Institutional (NIMHANS) Animal Ethics Committee, in accordance with the guidelines of the CPCSEA, India, and NIH, USA.

Consent for publication: Not applicable

Availability of data and materials: The raw datasets used and/or analysed during the current study are available from the corresponding author on reasonable request.

Competing interests: None of the authors have any conflict of interest.

Funding: The study was funded by DBT (No.BT/PR12518/MED/30/1462/2014) to PAA. APL was a UGC SRF.

Authors' contributions: APL contributed to study design, performed experiments and collected data and provided $1^{\text {st }}$ draft; UB performed experiments and collected data; MP performed statistical analysis of the data; RSK \& BKCS performed electron microscopy and data analysis; TRR and BMK: contributed to data analysis and revision of MS; PAA conceptualized the study, performed data analysis, edited the manuscript and obtained funds,.

Acknowledgements: We thank Dr. M.M. Srinivas Bharath, Head, Department of Clinical Psychopharmacology and Neurotoxicology; Dr. Gayathri N, Department of Neuropathology; Dr. Monojit Debnath, Department of Human Genetics, NIMHANS for laboratory facilities.

\section{References}

Abbas MM, Xu Z, Tan LCS.Epidemiology of Parkinson's Disease-East Versus West.Mov Disord Clin Pract. 2017 Dec 22;5(1):14-28. doi: 10.1002/mdc3.12568. eCollection 2018 Jan-Feb.

Alladi PA, Mahadevan A, Shankar SK, Raju TR, Muthane U. 2010a. Expression of GDNF receptors GFRalpha1 and RET is preserved in substantia nigra pars compacta of aging Asian Indians. J Chem Neuroanat. 2010 Sep;40(1):43-52. doi: 10.1016/j.jchemneu.2010.03.007. Epub 2010 Mar 27.

Alladi PA, Mahadevan A, Vijayalakshmi K, Muthane U, Shankar SK, Raju TR.2010b. Ageing enhances alpha-synuclein, ubiquitin and endoplasmic reticular stress protein expression in the nigral neurons of Asian Indians. Neurochem Int. 2010 Nov;57(5):530-9. doi: 10.1016/j.neuint.2010.06.018. Epub 2010 Jul 6.

Alladi PA, Mahadevan A, Yasha TC, Raju TR, Shankar SK, Muthane U. 2009. Absence of age-related changes in nigral dopaminergic neurons of Asian Indians: relevance to lower incidence of Parkinson's. Neuroscience. Mar 3;159(1):236-45. doi: 10.1016/j.neuroscience.2008.11.051.

Arimoto, T., Choi, D. Y., Lu, X., Liu, M., Nguyen, X. V., Zheng, N., ... Bing, G. (2007). Interleukin-10 protects against inflammation-mediated degeneration of dopaminergic neurons in substantia nigra. Neurobiology of Aging. Jun; 28(6): 894-906. https://doi.org/10.1016/j.neurobiolaging.2006.04.011

Loading [MathJax]/jax/output/CommonHTML/fonts/TeX/fontdata.js 
Balasingam, V., \& Yong, V. W. (1996). Attenuation of astroglial reactivity by interleukin-10. Journal of Neuroscience. May 1; $16(9): 2945-55$. https://doi.org/10.1523/jneurosci.16-09-02945.1996

Bernheimer, H., Birkmayer, W., Hornykiewicz, O., Jellinger, K., \& Seitelberger, F. (1973). Brain dopamine and the syndromes of Parkinson and Huntington Clinical, morphological and neurochemical correlations. Journal of the Neurological Sciences. Dec; 20(4): 415-55. https://doi.org/10.1016/0022-510X(73)90175-5

Bisht, K., Sharma, K. P., Lecours, C., Gabriela Sánchez, M., El Hajj, H., Milior, G., ... Tremblay, M. È. (2016). Dark microglia: A new phenotype predominantly associated with pathological states. GLIA. May 1; 64(5): 826-839. https://doi.org/10.1002/glia.22966

Blum-Degena, D., Müller, T., Kuhn, W., Gerlach, M., Przuntek, H., \& Riederer, P. (1995). Interleukin-1 $\beta$ and interleukin-6 are elevated in the cerebrospinal fluid of Alzheimer's and de novo Parkinson's disease patients. Neuroscience Letters. Dec 29; 202(1-2): 17-20. https://doi.org/10.1016/0304-3940(95)12192-7

Boje, K. M., \& Arora, P. K. (1992). Microglial-produced nitric oxide and reactive nitrogen oxides mediate neuronal cell death. Brain Research. Aug 7; 587(2): 2506. https://doi.org/10.1016/0006-8993(92)91004-X

Bok, E., Cho, E. J., Chung, E. S., Shin, W. H., \& Jin, B. K. (2018). Interleukin-4 contributes to degeneration of dopamine neurons in the lipopolysaccharidetreated substantia nigra in vivo. Experimental Neurobiology. Aug; 27(4): 309-319. https://doi.org/10.5607/en.2018.27.4.309

Boka, G., Anglade, P., Wallach, D., Javoy-Agid, F., Agid, Y., \& Hirsch, E. C. (1994). Immunocytochemical analysis of tumor necrosis factor and its receptors in Parkinson's disease. Neuroscience Letters. May 19; 172(1-2): 151-4. https://doi.org/10.1016/0304-3940(94)90684-X

Bolin, L. M., Strycharska-Orczyk, I., Murray, R., Langston, J. W., \& Di Monte, D. (2002). Increased vulnerability of dopaminergic neurons in MPTP-lesioned interleukin-6 deficient mice. Journal of Neurochemistry. Oct; 83(1): 167-75. https://doi.org/10.1046/j.1471-4159.2002.01131.x

Cardona, A. E., Pioro, E. P., Sasse, M. E., Kostenko, V., Cardona, S. M., Dijkstra, I. M., ... Ransohoff, R. M. (2006). Control of microglial neurotoxicity by the fractalkine receptor. Nature Neuroscience. Jul; 9(7): 917-24. https://doi.org/10.1038/nn1715

Casano, A. M., Albert, M., \& Peri, F. (2016). Developmental Apoptosis Mediates Entry and Positioning of Microglia in the Zebrafish Brain. Cell Reports. Jul 26; 16(4): 897-906. https://doi.org/10.1016/j.celrep.2016.06.033

Chao, C. C., Hu, S. X., Ehrlich, L., \& Peterson, P. K. (1995). Interleukin-1 and tumor necrosis factor-a synergistically mediate neurotoxicity: Involvement of nitric oxide and of n-methyl-d-aspartate receptors. Brain Behavior and Immunity. Dec; 9(4): 355-65. https://doi.org/10.1006/brbi.1995.1033

Chaudhari, S. N., \& Kipreos, E. T. (2017). Increased mitochondrial fusion allows the survival of older animals in diverse C. Elegans longevity pathways. Nature Communications. Aug 3; 8(1): 182. https://doi.org/10.1038/s41467-017-00274-4

Chaudhuri, K. R., Healy, D. G., \& Schapira, A. H. V. (2006). Non-motor symptoms of Parkinson's disease: Diagnosis and management. Lancet Neurology. Mar; 5(3): 235-45. https://doi.org/10.1016/S1474-4422(06)70373-8

Chertoff, M., Di Paolo, N., Schoeneberg, A., Depino, A., Ferrari, C., Wurst, W., ... Pitossi, F. (2011). Neuroprotective and neurodegenerative effects of the chronic expression of tumor necrosis factor a in the nigrostriatal dopaminergic circuit of adult mice. Experimental Neurology. Feb; $227(2): 237-51$.

https://doi.org/10.1016/j.expneurol.2010.11.010

Chhor, V., Le Charpentier, T., Lebon, S., Oré, M. V., Celador, I. L., Josserand, J., ... Fleiss, B. (2013). Characterization of phenotype markers and neuronotoxic potential of polarised primary microglia In vitro. Brain, Behavior, and Immunity. Aug; 32: 75-80. https://doi.org/10.1016/j.bbi.2013.02.005

Colla, E., Coune, P., Liu, Y., Pletnikova, O., Troncoso, J. C., Iwatsubo, T., ... Lee, M. K. (2012). Endoplasmic reticulum stress is important for the manifestations of a-synucleinopathy in vivo. Journal of Neuroscience. Mar 7; 32(10): 3306-20. https://doi.org/10.1523/JNEUR0SCI.5367-11.2012

Damani, M. R., Zhao, L., Fontainhas, A. M., Amaral, J., Fariss, R. N., \& Wong, W. T. (2011). Age-related alterations in the dynamic behavior of microglia. Aging Cell. Apr; 10(2): 263-76. https://doi.org/10.1111/j.1474-9726.2010.00660.x

Damier, P., Hirsch, E. C., Agid, Y., \& Graybiel, A. M. (1999). The substantia nigra of the human brain: Il. Patterns of loss of dopamine-containing neurons in Parkinson's disease. Brain. Aug; 122(Pt 8): 1437-48. https://doi.org/10.1093/brain/122.8.1437

Damier, Philippe, Kastner, A., Agid, Y., \& Hirsch, E. C. (1996). Does monoamine oxidase type B play a role in dopaminergic nerve cell death in Parkinson's disease? Neurology. May; 46(5): 1262-9. https://doi.org/10.1212/wnl.46.5.1262

Doré, S., Takahashi, M., Ferris, C. D., Hester, L. D., Guastella, D., \& Snyder, S. H. (1999). Bilirubin, formed by activation of heme oxygenase-2, protects neurons against oxidative stress injury. Proceedings of the National Academy of Sciences of the United States of America. Mar 2; 96(5): 2445-50.

https://doi.org/10.1073/pnas.96.5.2445

Dwyer, B. E., Nishimura, R. N., \& Lu, S. Y. (1995). Differential expression of heme oxygenase-1 in cultured cortical neurons and astrocytes determined by the aid of a new heme oxygenase antibody. Response to oxidative stress. Molecular Brain Research. May; 30(1): 37-47. https://doi.org/10.1016/0169-

$328 \times(94) 00273-\mathrm{H}$

Loading [MathJax]/jax/output/CommonHTML/fonts/TeX/fontdata.js 
Erny, D., De Angelis, A. L. H., Jaitin, D., Wieghofer, P., Staszewski, O., David, E., ... Prinz, M. (2015). Host microbiota constantly control maturation and function of microglia in the CNS. Nature Neuroscience. Jul; 18(7): 965-77. https://doi.org/10.1038/nn.4030

Farkas E, De Jong GI, de Vos RA, Jansen Steur EN, Luiten PG (2000) Pathological features of cerebral cortical capillaries are doubled in Alzheimer's disease and Parkinson's diseaseActa Neuropathol. Oct;100(4):395-402.

Ferrari, C. C., Pott Godoy, M. C., Tarelli, R., Chertoff, M., Depino, A. M., \& Pitossi, F. J. (2006). Progressive neurodegeneration and motor disabilities induced by chronic expression of IL-1 $\beta$ in the substantia nigra. Neurobiology of Disease. Oct-24(1): 183-93. https://doi.org/10.1016/j.nbd.2006.06.013

Fiebig, C., Keiner, S., Ebert, B., Schäffner, I., Jagasia, R., Lie, D. C., \& Beckervordersandforth, R. (2019). Mitochondrial dysfunction in astrocytes impairs the generation of reactive astrocytes and enhances neuronal cell death in the cortex upon photothrombotic lesion. Frontiers in Molecular Neuroscience. Feb 22; https://doi.org/10.3389/fnmol.2019.00040

Franceschi, C., Bonafè, M., Valensin, S., Olivieri, F., De Luca, M., Ottaviani, E., \&De Benedictis, G. (2006). Inflamm-aging: an evolutionary perspective on immunosenescence. Annals of the new york academy of sciences. Jan 25. Https://doi.org/10.1111/j.1749-6632.2000.tb06651.x

Gourie-Devi M. 2014. Epidemiology of neurological disorders in India: review of background, prevalence and incidence of epilepsy, stroke, Parkinson's disease and tremors.Neurol India. 2014 Nov-Dec;62(6):588-98. doi: 10.4103/0028-3886.149365. Review. Erratum in: Neurol India. 2016 Sep-0ct;64(5):1110-1.

Grabert, K., Michoel, T., Karavolos, M. H., Clohisey, S., Kenneth Baillie, J., Stevens, M. P., ... McColl, B. W. (2016). Microglial brain regionâ 'dependent diversity and selective regional sensitivities to aging. Nature Neuroscience. Mar; 19(3): 504-16. https://doi.org/10.1038/nn.4222

Grondin, R., Cass, W. A., Zhang, Z., Stanford, J. A., Gash, D. M., \& Gerhardt, G. A. (2003). Glial cell line-derived neurotrophic factor increases stimulus-evoked dopamine release and motor speed in aged rhesus monkeys. Journal of Neuroscience. Mar 1; 23(5): 1974-80. https://doi.org/10.1523/jneurosci.23-0501974.2003

Guerra, F., Girolimetti, G., Beli, R., Mitruccio, M., Pacelli, C., Ferretta, A., Gasparre G, Cocco T, Bucci, C. (2019). Synergistic Effect of Mitochondrial and Lysosomal Dysfunction in Parkinson's Disease. Cells. May 14; 8(5). pii; E 452. https://doi.org/10.3390/cells8050452

Heijtz, R. D., Wang, S., Anuar, F., Qian, Y., Björkholm, B., Samuelsson, A., ... Pettersson, S. (2011). Normal gut microbiota modulates brain development and behavior. Proceedings of the National Academy of Sciences of the United States of America. Feb 15; 8(7): 3047-3052.

https://doi.org/10.1073/pnas.1010529108

Hirsch, E. C., \& Hunot, S. (2009, April). Neuroinflammation in Parkinson's disease: a target for neuroprotection? The Lancet Neurology, Apr; 8(4) $382-97$. https://doi.org/10.1016/S1474-4422(09)70062-6

Hoekstra, J. G., Cook, T. J., Stewart, T., Mattison, H., Dreisbach, M. T., Hoffer, Z. S., \& Zhang, J. (2015). Astrocytic dynamin-like protein 1 regulates neuronal protection against excitotoxicity in Parkinson disease. American Journal of Pathology. Feb; 185(2): 536-49. https://doi.org/10.1016/j.ajpath.2014.10.022

Hofmann, K. W., Schuh, A. F. S., Saute, J., Townsend, R., Fricke, D., Leke, R., ... Rieder, C. R. M. (2009). Interleukin-6 serum levels in patients with parkinson's disease. Neurochemical Research. Aug; 34(8): 1401-4. https://doi.org/10.1007/s11064-009-9921-z

Hung, S. Y., Liou, H. C., Kang, K. H., Wu, R. M., Wen, C. C., \& Fu, W. M. (2008). Overexpression of heme oxygenase-1 protects dopaminergic neurons against 1 methyl-4-phenylpyridinium-induced neurotoxicity. Molecular Pharmacology. Dec; 74(6): 1564-75. https://doi.org/10.1124/mol.108.048611

Irwin, I., Delanney, L., Chan, P., Sandy, M. S., Monte, D. A. D., \& Langston, J. W. (1997). Nigrostriatal monoamine oxidase A and B in aging squirrel monkeys and C57BL/6 mice. Neurobiology of Aging. Mar-Apr; 18(2): 235-41. https://doi.org/10.1016/S0197-4580(97)00003-1

Jackson-Lewis, V., \& Przedborski, S. (2007). Protocol for the MPTP mouse model of Parkinson's disease. Nature Protocols. 2(1): 141-51. https://doi.org/10.1038/nprot.2006.342

Jeohn, G. H., Kong, L. Y., Wilson, B., Hudson, P., \& Hong, J. S. (1998). Synergistic neurotoxic effects of combined treatments with cytokines in murine primary mixed neuron/glia cultures. Journal of Neuroimmunology. May 1; 85(1): 1-10. https://doi.org/10.1016/S0165-5728(97)00204-X

Jyothi, H. J., Vidyadhara, D. J., Mahadevan, A., Philip, M., Parmar, S. K., Manohari, S. G., ... Alladi, P. A. (2015). Aging causes morphological alterations in astrocytes and microglia in human substantia nigra pars compacta. Neurobiology of Aging, Dec; 36(12), 3321-3333.

https://doi.org/10.1016/j.neurobiolaging.2015.08.024

Katoh, M., Wu, B., Nguyen, H. B., Thai, T. Q., Yamasaki, R., Lu, H., ... Ohno, N. (2017). Polymorphic regulation of mitochondrial fission and fusion modifies phenotypes of microglia in neuroinflammation. Scientific Reports. Jul 10; 7(1): 4942. https://doi.org/10.1038/s41598-017-05232-0

Kelly, J. R., Kennedy, P. J., Cryan, J. F., Dinan, T. G., Clarke, G., \& Hyland, N. P. (2015). Breaking down the barriers: The gut microbiome, intestinal permeability and stress-related psychiatric disorders. Frontiers in Cellular Neuroscience. Oct 14; 9(392). https://doi.org/10.3389/fncel.2015.00392

Kim, W. G., Mohney, R. P., Wilson, B., Jeohn, G. H., Liu, B., \& Hong, J. S. (2000). Regional difference in susceptibility to lipopolysaccharide-induced neurotoxicity in the rat brain: Role of microglia. Journal of Neuroscience. Aug 15; 20(16): 6309-16. https://doi.org/10.1523/jneurosci.20-16-06309.2000

Loading [MathJax]/jax/output/CommonHTML/fonts/TeX/fontdata.js

Page $13 / 24$ 
Kohutnicka, M., Lewandowska, E., Kurkowska-Jastrz囚bska, I., Członkowski, A., \& Członkowska, A. (1998). Microglial and astrocytic involvement in a murine model of Parkinson's disease induced by 1-methyl-4-phenyl-1,2,3,6-tetrahydropyridine (MPTP). Immunopharmacology. Jun; 39(3): 167-80.

https://doi.org/10.1016/S0162-3109(98)00022-8

Lawson, L. J., Perry, V. H., Dri, P., \& Gordon, S. (1990). Heterogeneity in the distribution and morphology of microglia in the normal adult mouse brain. Neuroscience. 39(1): 151-70. https://doi.org/10.1016/0306-4522(90)90229-W

Ledeboer, A., Brevé, J. J. P., Wierinckx, A., Van Der Jagt, S., Bristow, A. F., Leysen, J. E., ... Van Dam, A. M. (2002). Expression and regulation of interleukin-10 and interleukin-10 receptor in rat astroglial and microglial cells. European Journal of Neuroscience. Oct; 16(7): 1175-85. https://doi.org/10.1046/j.14609568.2002.02200.x

Lee, I., Tiwari, N., Dunlop, M. H., Graham, M., Liu, X., \& Rothman, J. E. (2014). Membrane adhesion dictates Golgi stacking and cisternal morphology. Proceedings of the National Academy of Sciences of the United States of America. Feb 4; 111(5): 1849-54 . https://doi.org/10.1073/pnas.1323895111

Lewis, T. L., Kwon, S. K., Lee, A., Shaw, R., \& Polleux, F. (2018). MFF-dependent mitochondrial fission regulates presynaptic release and axon branching by limiting axonal mitochondria size. Nature Communications. https://doi.org/10.1038/s41467-018-07416-2

Lyons, A., McQuillan, K., Deighan, B. F., O’Reilly, J. A., Downer, E. J., Murphy, A. C., ... Lynch, M. A. (2009). Decreased neuronal CD200 expression in IL-4-deficient mice results in increased neuroinflammation in response to lipopolysaccharide. Brain, Behavior, and Immunity. Oct; 23(7): $1020-7$.

https://doi.org/10.1016/j.bbi.2009.05.060

McGeer, P. L., Itagaki, S., Boyes, B. E., \& McGeer, E. G. (1988). Reactive microglia are positive for HLA-DR in the: Substantia nigra of Parkinson's and Alzheimer's disease brains. Neurology. Aug; 38(8): 1285-91. https://doi.org/10.1212/wnl.38.8.1285

Mogi, M., Harada, M., Riederer, P., Narabayashi, H., Fujita, K., \& Nagatsu, T. (1994). Tumor necrosis factor-a (TNF-a) increases both in the brain and in the cerebrospinal fluid from parkinsonian patients. Neuroscience Letters. Jan 3; 165(1-2): 208-10. https://doi.org/10.1016/0304-3940(94)90746-3

Montagne, A., Barnes, S. R., Sweeney, M. D., Halliday, M. R., Sagare, A. P., Zhao, Z., ... Zlokovic, B. V. (2015). Blood-Brain barrier breakdown in the aging human hippocampus. Neuron. Jan 21; 85(2): 296-302 https://doi.org/10.1016/j.neuron.2014.12.032

Nathan, C., \& Xie, Q. wen. (1994). Nitric oxide synthases: Roles, tolls, and controls. Cell. Sept 23; 78(6): 915-8. https://doi.org/10.1016/0092-8674(94)90266-6

Noh, H., Jeon, J., \& Seo, H. (2014). Systemic injection of LPS induces region-specific neuroinflammation and mitochondrial dysfunction in normal mouse brain. Neurochemistry International. Apr; 69: 35-40. https://doi.org/10.1016/j.neuint.2014.02.008

Petrache, I., Otterbein, L. E., Alam, J., Wiegand, G. W., \& Choi, A. M. K. (2000). Heme oxygenase-1 inhibits TNF-a-induced apoptosis in cultured fibroblasts. American Journal of Physiology - Lung Cellular and Molecular Physiology. Feb; 278(2): L312-9. https://doi.org/10.1152/ajplung.2000.278.2.I312

Pintado, C., Revilla, E., Vizuete, M. L., Jiménez, S., García-Cuervo, L., Vitorica, J., ... Castaño, A. (2011). Regional difference in inflammatory response to LPSinjection in the brain: Role of microglia cell density. Journal of Neuroimmunology. Sep 15; 238(1-2) : 44-51. https://doi.org/10.1016/j.jneuroim.2011.06.017

Rabey, J. M., Sagi, I., Huberman, M., Melamed, E., Korczyn, A., Giladi, N., ... Berecz, G. (2000). Rasagiline mesylate, a new MAO-B inhibitor for the treatment of Parkinson's disease: A double-blind study as adjunctive therapy to levodopa. Clinical Neuropharmacology. Nov-Dec; 23(6): 324-30.

https://doi.org/10.1097/00002826-200011000-00005

Scheperjans, F., Aho, V., Pereira, P. A. B., Koskinen, K., Paulin, L., Pekkonen, E., ... Auvinen, P. (2015). Gut microbiota are related to Parkinson's disease and clinical phenotype. Movement Disorders. Mar;30(3):350-8. https://doi.org/10.1002/mds.26069

Schipper, H. M., Liberman, A., \& Stopa, E. G. (1998). Neural heme oxygenase-1 expression in idiopathic Parkinson's disease. Experimental Neurology.Mar;150(1):60-8. https://doi.org/10.1006/exnr.1997.6752

Seshadri, A., \& Alladi, P. A. (2019). Divergent Expression Patterns of Drp1 and HSD10 in the Nigro-Striatum of Two Mice Strains Based on their MPTP Susceptibility. Neurotoxicity Research. Apr 16; 36(1):27-38. https://doi.org/10.1007/s12640-019-00036-8

Smeyne, M., Goloubeva, O., \& Smeyne, R. J. (2001). Strain-dependent susceptibility to MPTP and MPP+-induced parkinsonism is determined by glia. GLIA. Apr 15;34(2):73-80. https://doi.org/10.1002/glia.1042

Snow, B. J., Rolfe, F. L., Lockhart, M. M., Frampton, C. M., O’Sullivan, J. D., Fung, V., ... Taylor, K. M. (2010). A double-blind, placebo-controlled study to assess the mitochondria- targeted antioxidant MitoQ as a disease-modifying therapy in Parkinson's disease. Movement Disorders. Aug 15;25(11):1670-4. https://doi.org/10.1002/mds.23148

Song, W., Kothari, V., Velly, A. M., Cressatti, M., Liberman, A., Gornitsky, M., \& Schipper, H. M. (2018). Evaluation of salivary heme oxygenase-1 as a potential biomarker of early Parkinson's disease. Movement Disorders. Apr;33(4):583-591. https://doi.org/10.1002/mds.27328

Soreq, L., Rose, J., Soreq, E., Hardy, J., Trabzuni, D., Cookson, M. R., ... Ule, J. (2017). Major Shifts in Glial Regional Identity Are a Transcriptional Hallmark of Human Brain Aging. Cell Reports. Jan 10;18(2):557-570. https://doi.org/10.1016/j.celrep.2016.12.011

Loading [MathJax]/jax/output/CommonHTML/fonts/TeX/fontdata.js

Page $14 / 24$ 
Spindler, M., Flint Beal, M., \& Henchcliffe, C. (2009). Coenzyme Q10 effects in neurodegenerative disease. Neuropsychiatric Disease and Treatment. Nov 16; 5: 597-610. https://doi.org/10.2147/ndt.s5212

Sriram, K., Miller, D. B., \& O'Callaghan, J. P. (2006). Minocycline attenuates microglial activation but fails to mitigate striatal dopaminergic neurotoxicity: Role of tumor necrosis factor-a. Journal of Neurochemistry. Jan 9; 96(3): 706-718. https://doi.org/10.1111/j.1471-4159.2005.03566.x

Strickland, D., \& Bertoni, J. M. (2004). Parkinson's prevalence estimated by a state registry. Movement Disorders. Mar;19(3):318-23.

https://doi.org/10.1002/mds.10619

Tachikawa, M., \& Mochizuki, A. (2017). Golgi apparatus self-organizes into the characteristic shape via postmitotic reassembly dynamics. Proceedings of the National Academy of Sciences of the United States of America. May 16;114(20):5177-5182. https://doi.org/10.1073/pnas.1619264114

Turmaine, M., Raza, A., Mahal, A., Mangiarini, L., Bates, G. P., \& Davies, S. W. (2000). Nonapoptotic neurodegeneration in a transgenic mouse model of Huntington's disease. Proceedings of the National Academy of Sciences of the United States of America. Jul 5;97(14):8093-7.

https://doi.org/10.1073/pnas.110078997

Van Den Eeden SK, Tanner CM, Bernstein AL, Fross RD, Leimpeter A, Bloch DA, Nelson LM. 2003 Incidence of Parkinson's disease: variation by age, gender, and race/ethnicity. Am J Epidemiol. Jun 1;157(11):1015-22.

Vidyadhara, D. J., Yarreiphang, H., Raju, T. R., \& Alladi, P. A. (2017). Admixing of MPTP-Resistant and Susceptible Mice Strains Augments Nigrostriatal Neuronal Correlates to Resist MPTP-Induced Neurodegeneration. Molecular Neurobiology. Oct;54(8):6148-6162. https://doi.org/10.1007/s12035-016-0158-y

Vidyadhara DJ, Sasidharan A, Kutty BM, Raju TR, Alladi PA. 2019Admixing MPTP-resistant and MPTP-vulnerable mice enhances striatal field potentials and calbindin-D28K expression to avert motor behaviour deficits. Behav Brain Res. Mar 15; 360:216-227. doi: 10.1016/j.bbr.2018.12.015. Epub 2018 Dec 7.

Wirdefeldt, K., Adami, H. O., Cole, P., Trichopoulos, D., \& Mandel, J. (2011). Epidemiology and etiology of Parkinson's disease: A review of the evidence. European Journal of Epidemiology.Jun; 26 Suppl 1:S1-58. https://doi.org/10.1007/s10654-011-9581-6

Yim, N., Ryu, S. W., Han, E. C., Yoon, J., Choi, K., \& Choi, C. (2014). Mutant ubiquitin UBB+1 induces mitochondrial fusion by destabilizing mitochondrial fissionspecific proteins and confers resistance to oxidative stress-induced cell death in astrocytic cells. PLoS ONE. Jun 18;9(6):e99937.

https://doi.org/10.1371/journal.pone.0099937

Zhang, X., Du, L., Zhang, W., Yang, Y., Zhou, Q., \& Du, G. (2017). Therapeutic effects of baicalein on rotenone-induced Parkinson's disease through protecting mitochondrial function and biogenesis. Scientific Reports. Aug 30; 79968. https://doi.org/10.1038/s41598-017-07442-y

Zhao F, Wang W, Wang C, Siedlak SL, Fujioka H, Tang B, Zhu X. 2017 Mfn2 protects dopaminergic neurons exposed to paraquat both in vitro and in vivo: Implications for idiopathic Parkinson's disease.Biochim Biophys Acta Mol Basis Dis. Jun;1863(6):1359-1370. doi: 10.1016/j.bbadis.2017.02.016. Epub 2017 Feb 16.

\section{Figures}



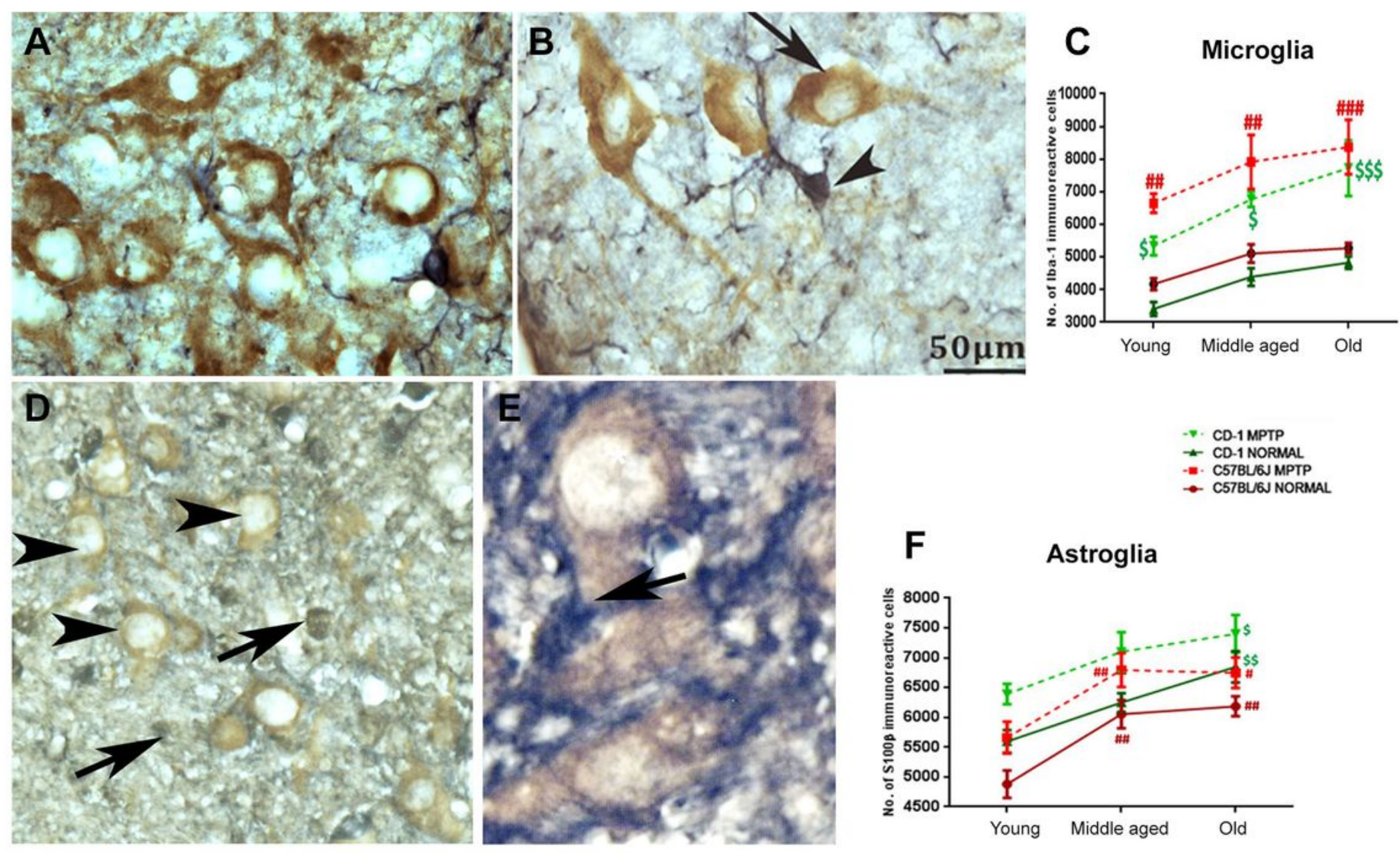

- CD-1 MPTP

* CD-1 NORMAL

- C57BL6J MPTP

- C57BLEL NORMAL

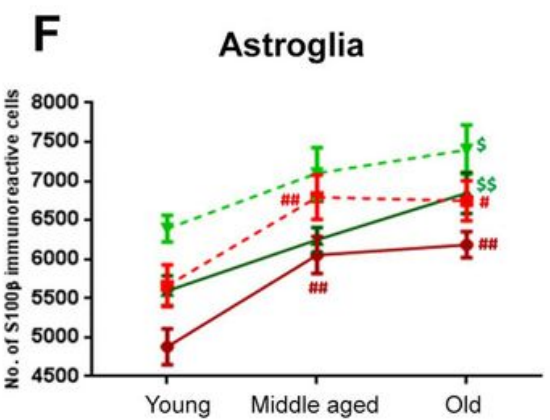

Figure 1

Baseline numbers of microglia and astrocytes differ in the two strains. More microglia in the susceptible strain: A\&B) Representative DAB stained photomicrographs of Iba-1 ir microglia (black) and TH positive DA neurons (brown) in SNpc. C) The histogram shows C57BL/6J had more Iba-1 ir cells than CD-1. Note the gradual increase in the microglial numbers during aging in both strains. Note the significant increase in the number of microglia at all ages after MPTP injection [Young (C57BL/6J normal vs. C57BL/6J MPTP \#\# p<0.01, CD-1 normal vs. CD-1 MPTP \# p<0.05); middle-aged (C57BL/6J normal vs. C57BL/6J MPTP \#\# p<0.01, CD-1 normal vs. CD-1 MPTP

$\mathrm{p}<0.01$ ); old (C57BL/6J normal vs. C57BL/6J MPTP \# \# \#p<0.001, CD-1 normal vs. CD-1 MPTP

$\$ p<0.001)$, (difference *between strains, \#within C57BL/6J,\$within CD-1). Scale bar: $50 \mu \mathrm{m}$. Fewer astrocytes in the susceptible strain: D\&E) Representative DAB stained photomicrographs of s100ß-ir astrocytes (black) and TH immunopositive DA neurons (brown) in SNpc. F) Note the significant increase in the numbers of astrocytes during aging in both strains and in response to MPTP. C57BL/6J normal (young vs. middle-aged \#\# p<0.01, young vs. old \#\# $p<0.01$ ), C57BL/6J MPTP (young vs. middle-aged \#\# $p<0.01$, young vs. old \# $p<0.05$ ), CD-1 normal (young vs. old $\$ \$ p<0.01$ ) CD-1 MPTP (young vs. old $\$ p<0.05$ ) (difference *between strains, \#within C57BL/6J, \$within CD-1). Scale bar: $50 \mu \mathrm{m}$. 
TNF $\alpha$
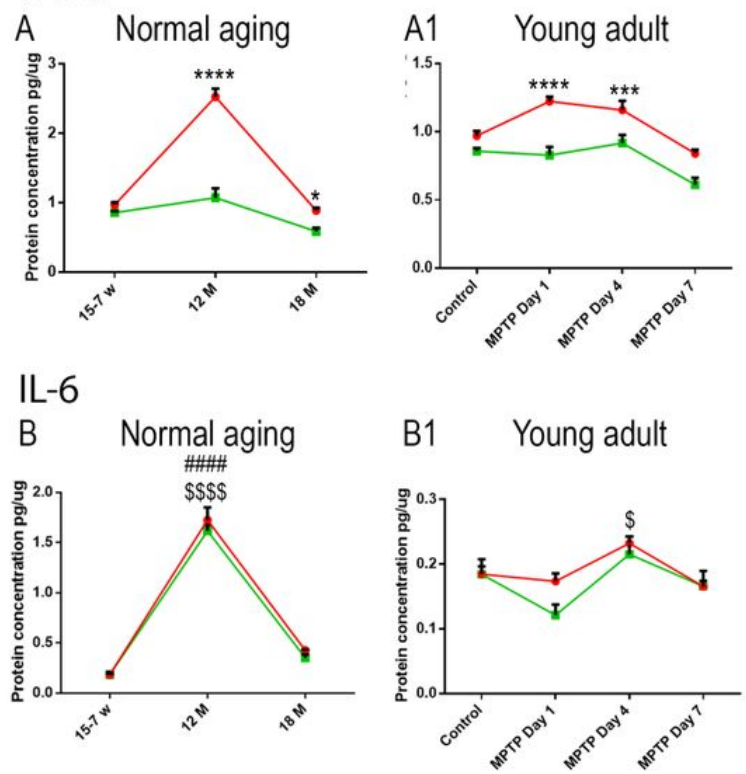

IL-1 $\beta$

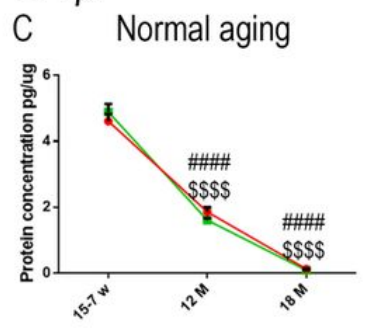

\section{Pro-inflammatory cytokines}
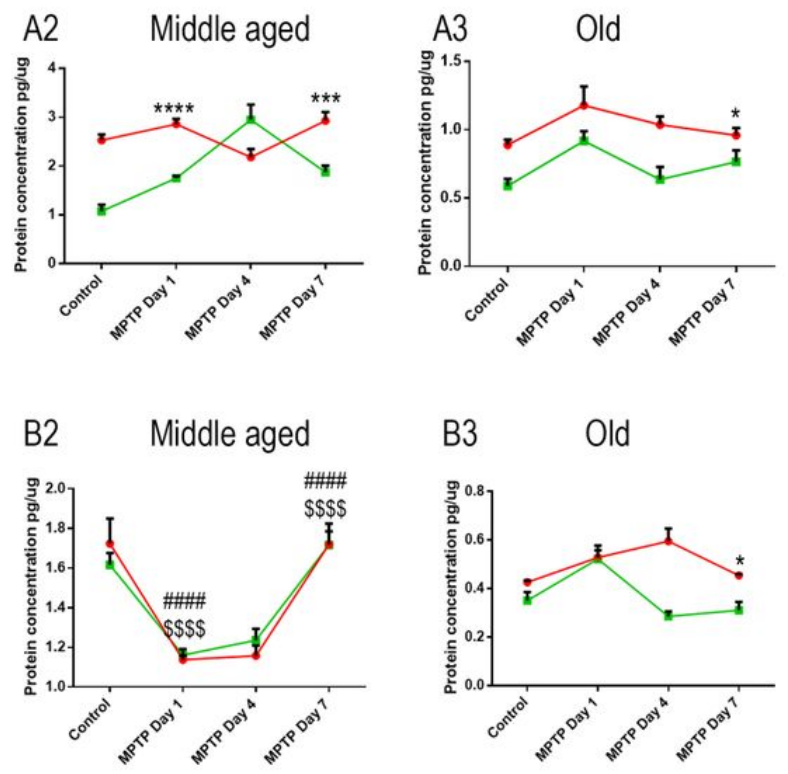

B3 Old
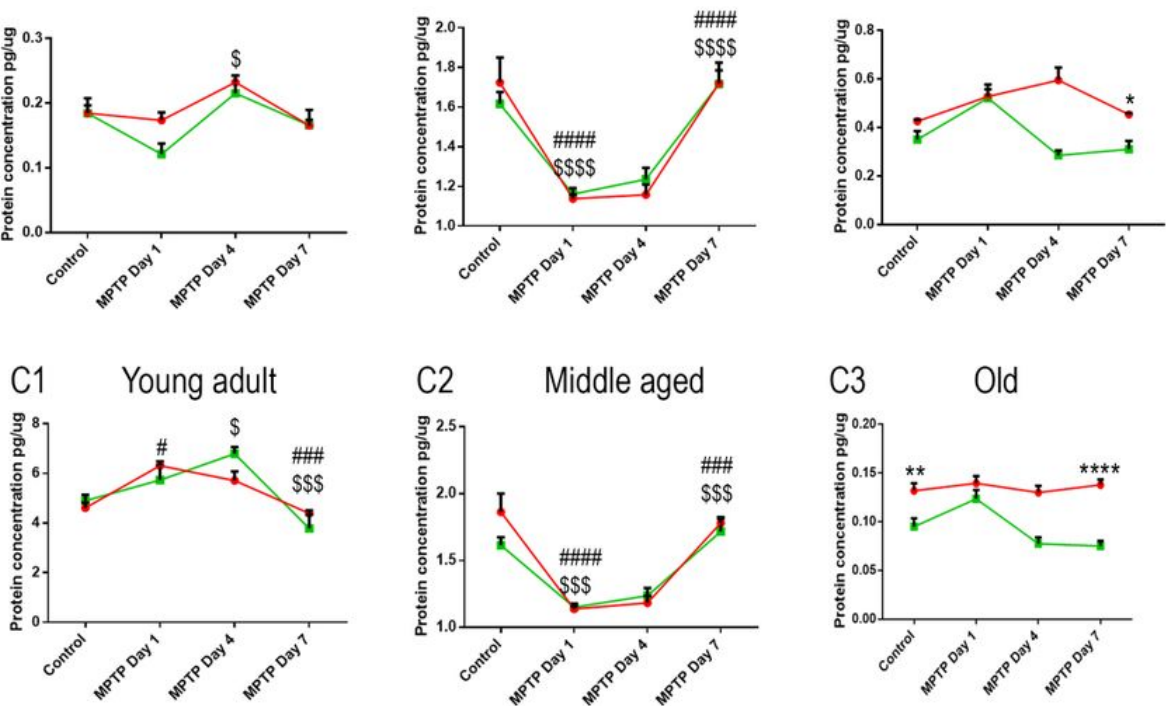

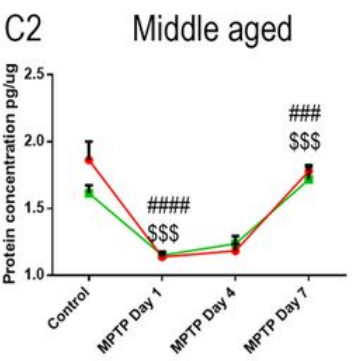

\section{Figure 2}

Levels of Pro-inflammatory cytokines A) Higher pro-inflammatory cytokine levels (TNF-a) in C57BL/6J: Note the significant increase in the basal level expression of TNF- $a$ in the middle-age C57BL/6J (C57BL/6J vs. CD-1 $* * \star * ~ p<0.0001)$. The level of pro-inflammatory cytokine TNF-a was significantly higher in old aged animals of $\mathrm{C} 57 \mathrm{BL} / 6 \mathrm{~J}$ compared to $\mathrm{CD}-1,{ }^{*} \mathrm{p}<0.05$. Upon MPTP injection C57BL/6J showed an upregulation in TNF- $\mathrm{a}$ expression at all the time points and age groups where as in CD-1 the changes were negligible. Young (C57BL/6J vs. CD-1, post-MPTP d1, *** p<0.001),young (C57BL/6J vs. CD-1, post-MPTP

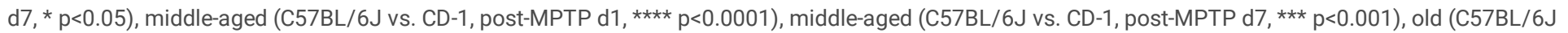
vs. CD-1, post-MPTP d4, * p 0 .05). B) Middle-aged animals showed significant increase in IL-6: Differences in the basal level expression of IL- 6 in different age groups were comparable between strains. Note the significant increase in the basal level expression of IL-6 in the middle-aged group. C57BL/6J [(young vs. middle-aged \#\#\# p<0.0001), (middle-aged vs. old \#\#\#\# p<0.001)] CD-1 [(young vs. middle-aged

$\mathrm{p}<0.0001)$, (middle-aged vs. old

p<0.0001)] and following MPTP CD-1 young (post-MPTP d1 vs. d4 \$ p<0.05), C57BL/6J middle-aged (control vs. post-MPTP day1 \#\#\#\# p<0.0001, post-MPTP d4 vs. d7 \#\#\#\# p<0.0001), CD-1 middle-aged (control vs. post-MPTP day1

p<0.001, post-MPTP d4 vs. d7

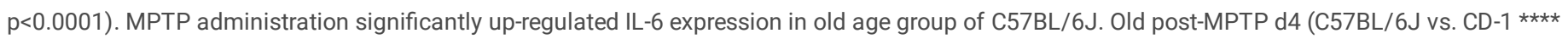
$\mathrm{p}<0.0001)$, post-MPTP day7 (C57BL/6J vs. CD-1 * $\mathrm{p}<0.05)$. C) IL-1 $\beta$ is down regulated during normal aging: Note the significant down-regulation in IL-1 $\beta$ expression during aging in both strains. C57BL/6J [(young vs. middle-aged \#\#\#\# p<0.0001), (middle-aged vs. old \#\#\#\# p<0.001)] CD-1 (young vs. middleaged

$\mathrm{p}<0.0001)$, (middle-aged vs. old

$\mathrm{p}<0.0001)]$. In MPTP treated animals, young adults and middle-aged groups showed significant difference at different time points. C57BL/6J young (control vs. post-MPTP day1 \# p<0.05, post-MPTP d1 vs. post-MPTP d7 \#\#\# p<0.001). CD-1 young (control vs. d4 \$ p<0.05), CD-1 young (d4 vs. d7 


\section{Anti-inflammatory cytokines}

\section{TGF- $\beta$}

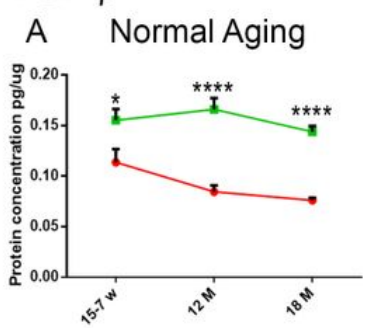

IL-4
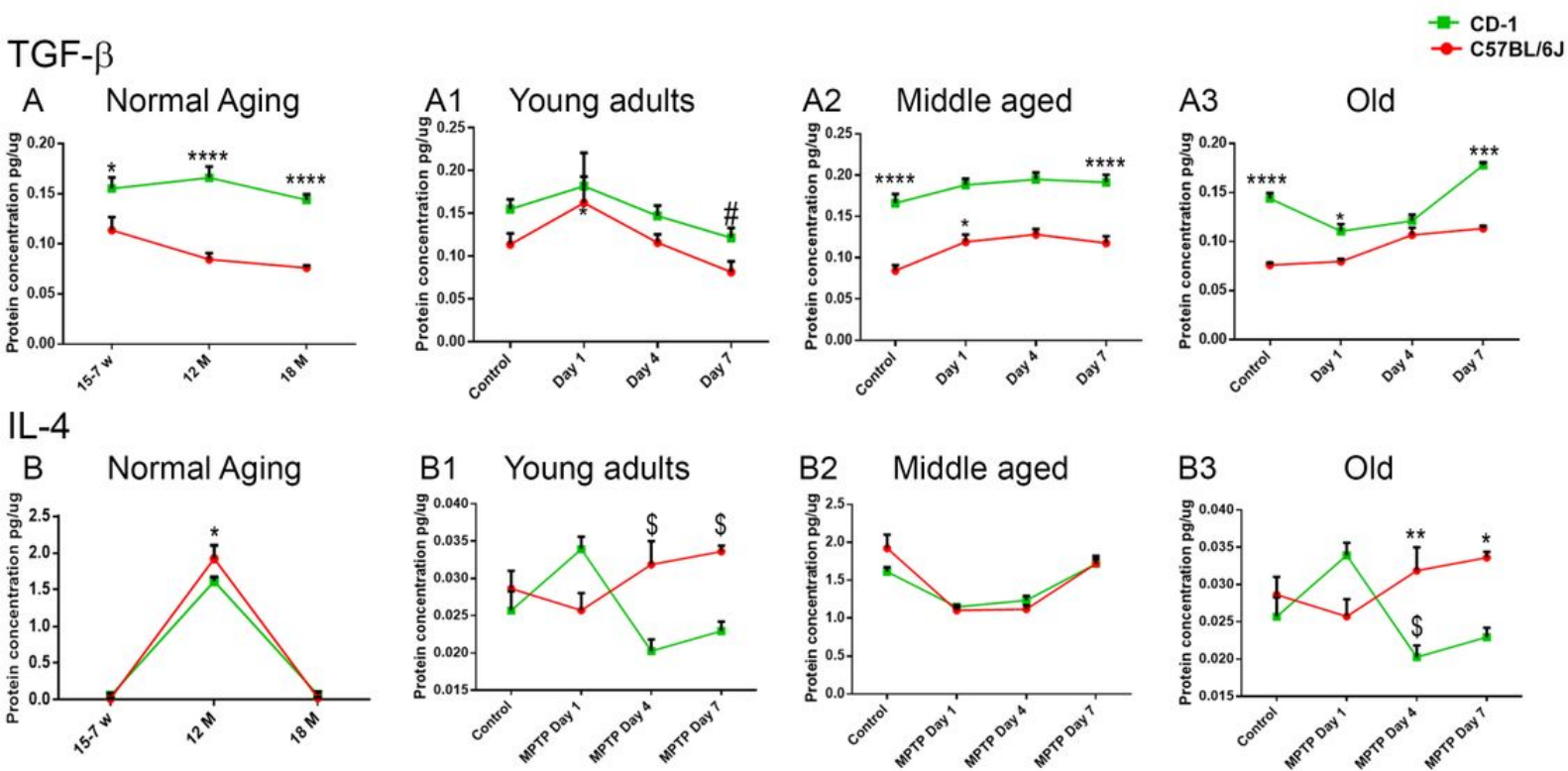

\section{IL-10}

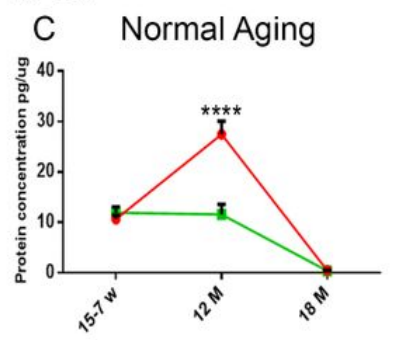

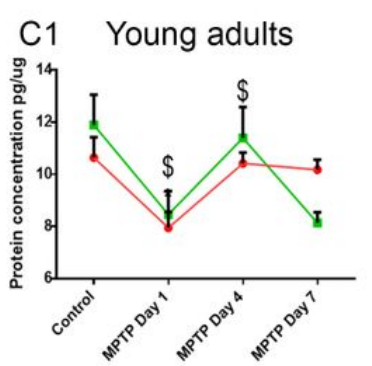
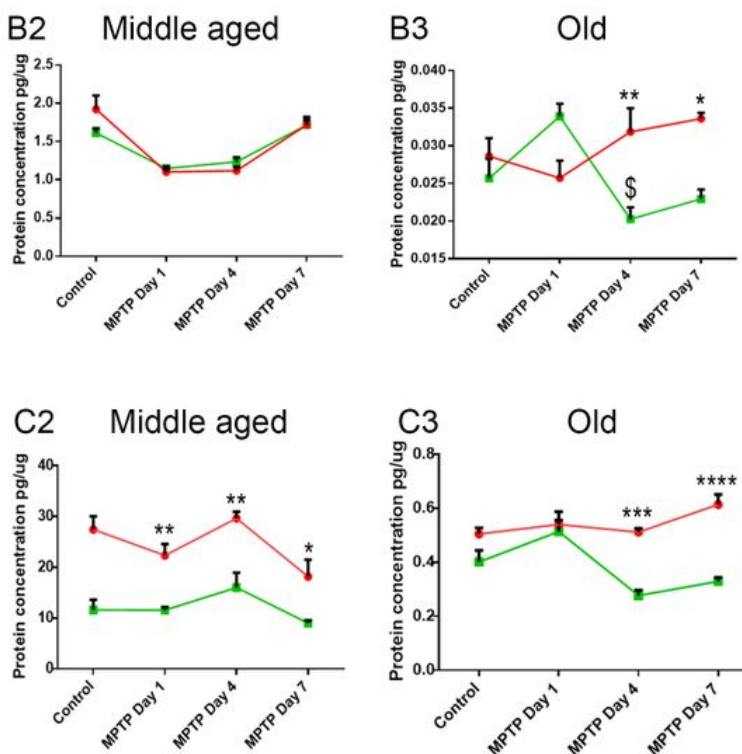

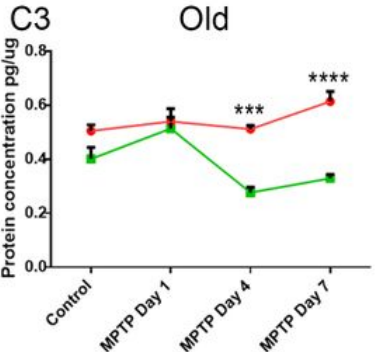

Figure 3

Levels of Anti-inflammatory cytokines A) Higher levels of anti-inflammatory cytokine TGF- $\beta$ in CD-1 mice: Note higher basal expression of anti-inflammatory TGF- $\beta$ in CD-1 compared to C57BL/6J in all three age groups (young, middle and old aged), young control (C57BL/6J vs. CD-1 *p<0.05), middle-aged control

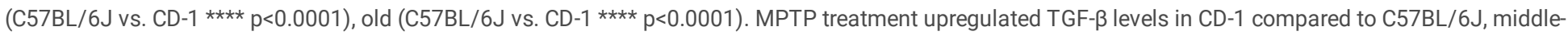

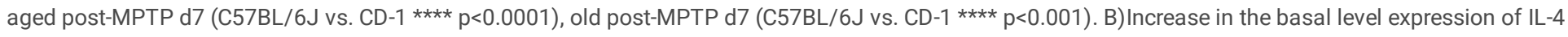
in middle-aged animals: Middle-aged C57BL/6J showed a significant increase compared to CD-1 (C57BL/6J vs. CD-1 * $p<0.05$ ). Upon MPTP administration old animals showed a significant increase in IL-4 expression of C57BL/6J, old post-MPTP d4 (C57BL/6J vs.CD-1 ** p<0.01), old post-MPTP d7 (C57BL/6J vs.CD-1 * p <0.05). C) Compensatory response by IL-10 in C57BL/6J: Note a significant increase in the basal expression of IL-10 in middle-age C57BL/6J,

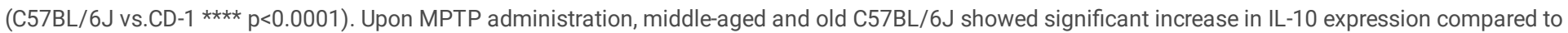
CD-1. Middle-aged post-MPTP d1\& 4 (C57BL/6J vs. CD-1 ** p<0.01), middle-aged post-MPTP day7 (C57BL/6J vs. CD-1 * p<0.05), old post-MPTP d4

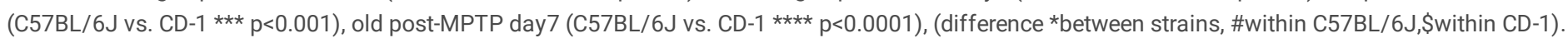



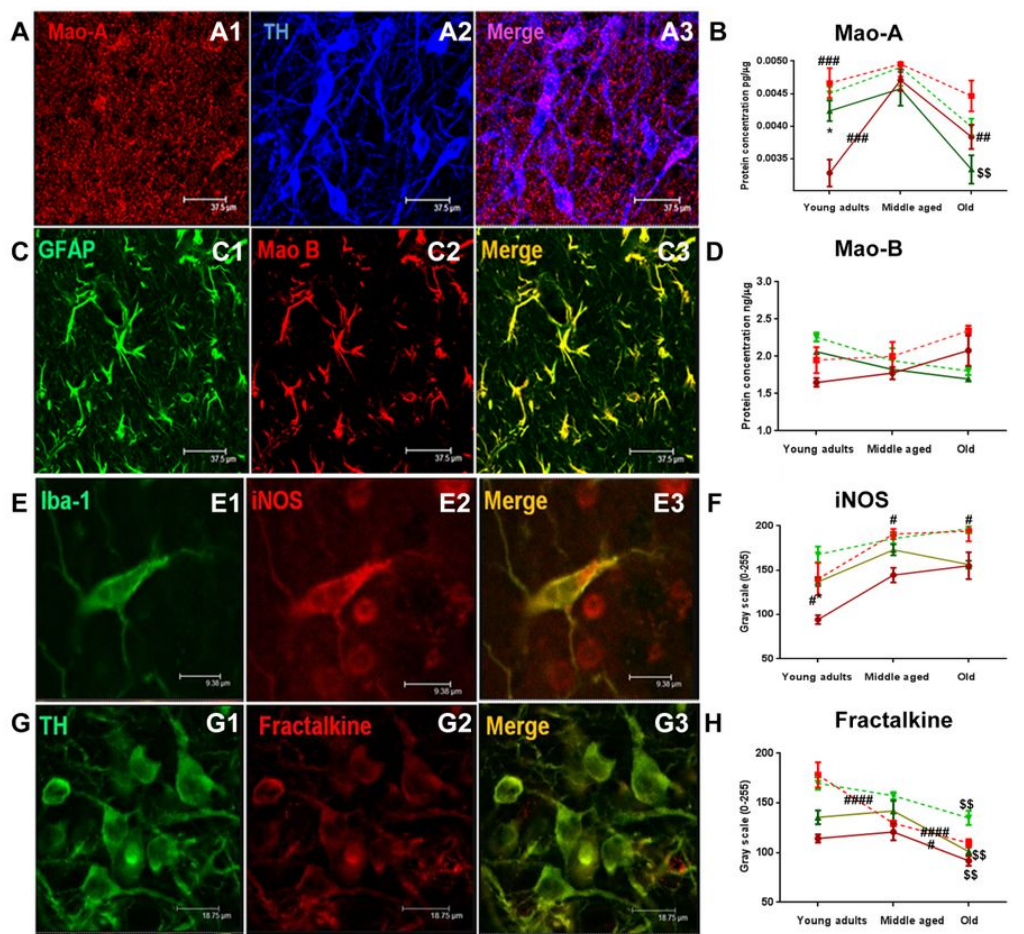

3
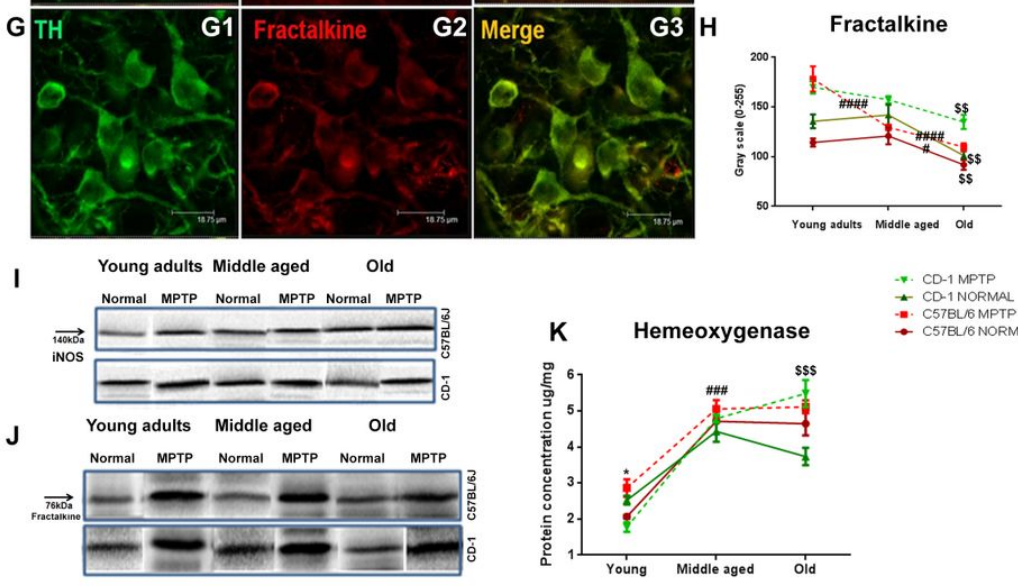

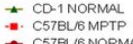

\section{Figure 4}

Essential Enzymes are differentially modulated in a strain specific manner: A) Representative photomicrographs of DA neurons (A1, TH, blue; cytoplasmic) colabeled with MAO-A (A2, red; punctate staining), and merge showing presence of MAO-A in dopaminergic neurons in SNpc (A3; pink). Note the punctate staining of MAO-A vis-a-vis the pan cytoplasmic staining of TH. B) Representative immunofluoresence photomicrographs of astrocytes (GFAP, green, B1) colabeled with MAO-B (red, B2) in striatum and the merge showing glial localization of MAO-B (B3, yellowish green). B\&D). The basal levels of MAO-A and MAO$B$ are higher in CD-1 (MAO-A C57BL/6J vs. CD-1 * $p<0.05$ ). Note the significant upregulation of MAO-A level in middle-aged C57BL/6J (young vs. middle-aged \#\#\# $\mathrm{p}<0.001)$. Aging increased the MAO-B levels in C57BL/6J whereas CD-1 showed a down regulation. MPTP enhanced MAO-A and MAO-B levels in both strains. (Difference *between strains, \#within C57BL/6J,\$within CD-1).E) Representative immunofluorescence photomicrographs of microglia (E1, Iba-1, green) co-labeled with iNOS (E2, red) and their co-labeling (E3, merge, yellowish orange) in SNpc. Note the presence of some INOS ir cells that were not Iba-1 ir. $(E 3$, red). F). Histograms show that basal iNOS level was significantly higher in CD-1 mice in young adults (C57BL/6J vs. CD-1 * p<0.05; I) I) Western blot showing a single band of iNOS at $140 \mathrm{kDa}$. Note the gradual increase at middle-age in both strains. MPTP caused a strain independent augmentation in all age groups (middle-aged C57BL/6J control vs. MPTP\# $p<0.05$, old C57BL/6J control vs. MPTP\# $p<0.05$, old CD- 1 control vs. MPTP\$ $p<0.05$. G) Representative immunofluorescence photomicrographs of DA neurons (G1, TH, green) co-labelled with fractalkine (G2, red) and their co-labeling (G3, merge, yellow-green) in SNpc. H) histograms show higher levels in CD-1 at all ages. Both strains show a gradual decrease with age (C57BL/6J middle-age vs. old $\# p<0.05, C D-1$ middle-age vs. old

$\mathrm{p}<0.01$ ). In C57BL/6J, the response to MPTP at middle and old age was lesser (young vs. middle-aged\#\#\#\#p<0.0001, middle-age vs. old \#\#\#\#p<0.0001).CL $\$ p<0.001)$. Both strains show increased expression after MPTP (C57BL/6J vs CD-1, young * $<<0.05$; CD-1 old control vs. MPTP\$\$ $p<0.001)$, (Difference *between strains, \#within C57BL/6J, \$ within CD-1). 


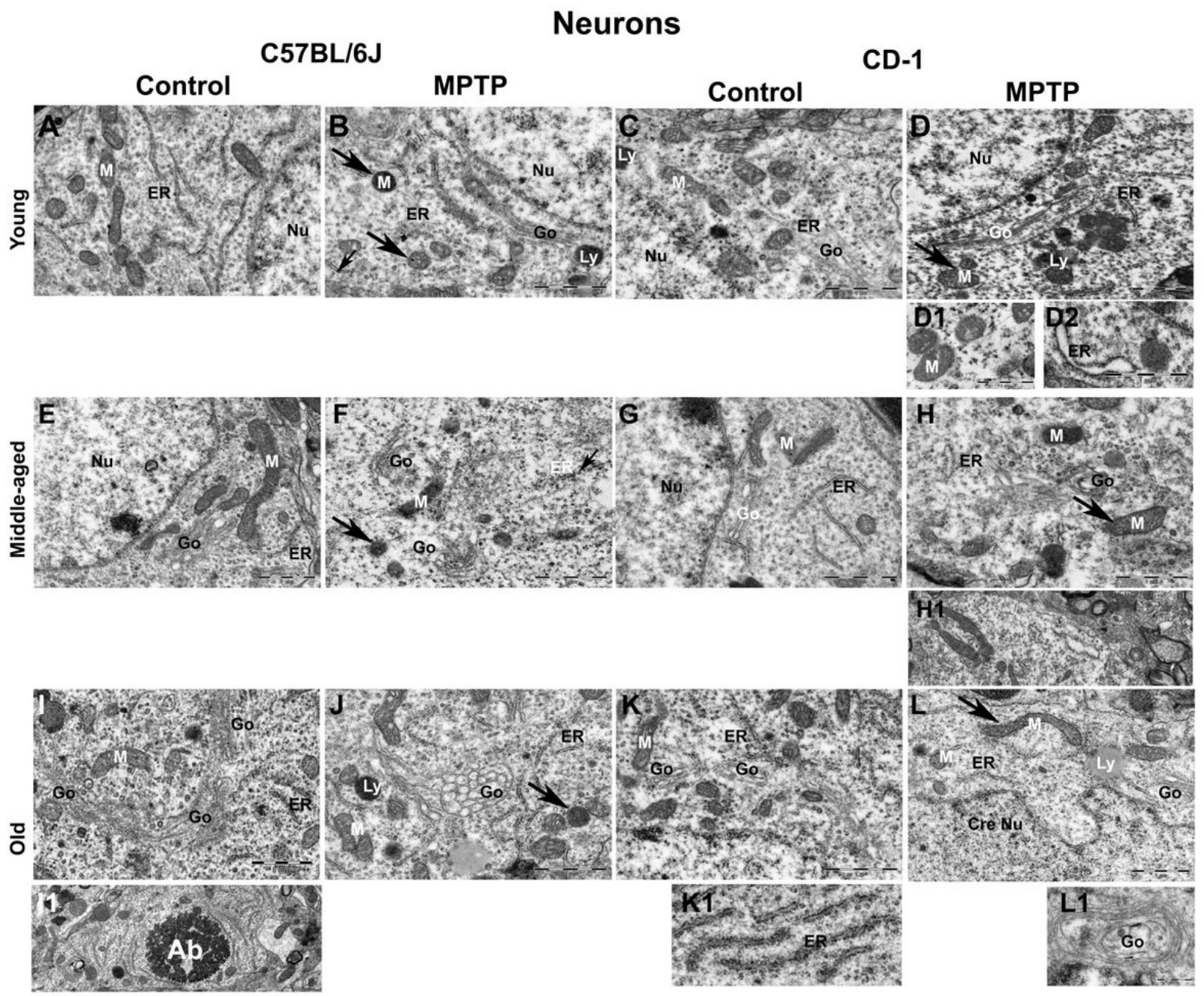

Figure 5

Age-related and MPTP-induced ultrastructural changes in substantia nigra neurons Representative electron micrographs of substantia nigra neurons at different ages and in response to MPTP. Note that the mitochondria of the elderly C57BL/6J were relatively shrunken (compare A,E\&I). MPTP induced mitochondrial shrinkage at all ages (compare A\&B and E\&F, I\&J; ' $M$ ' arrows). In CD-1 the mitochondrial size was fairly preserved with age (compare $C \& D$, D1

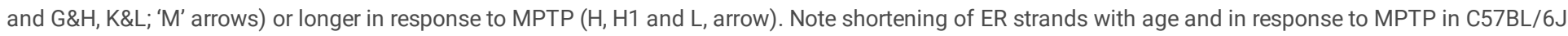
(compare A\&B and E\&F, I\&J; ER). Note the dilated ER in CD-1 neurons upon MPTP in young (D2) and at middle-age (ER, H \& H1). Note the ER arrays in aged CD-1 (K1) and presence of many Golgi units in middle aged and old mice of both strains ('Go' in I\&K). MPTP-administered aged C57BL/6J have circular Golgi with bloated saccules (J\&L1; 'Go'). Older C57BL/6J have apoptotic bodies (I1; 'Ab') and CD-1 has crenellated neuronal nucleus. Note several lysosomes in young MPTP injected CD-1 against few in C57BL/6J (B\&D 'Ly'). The scale bar for A=B, and others are specified for each photomicrograph. 


\section{Astrocytes}

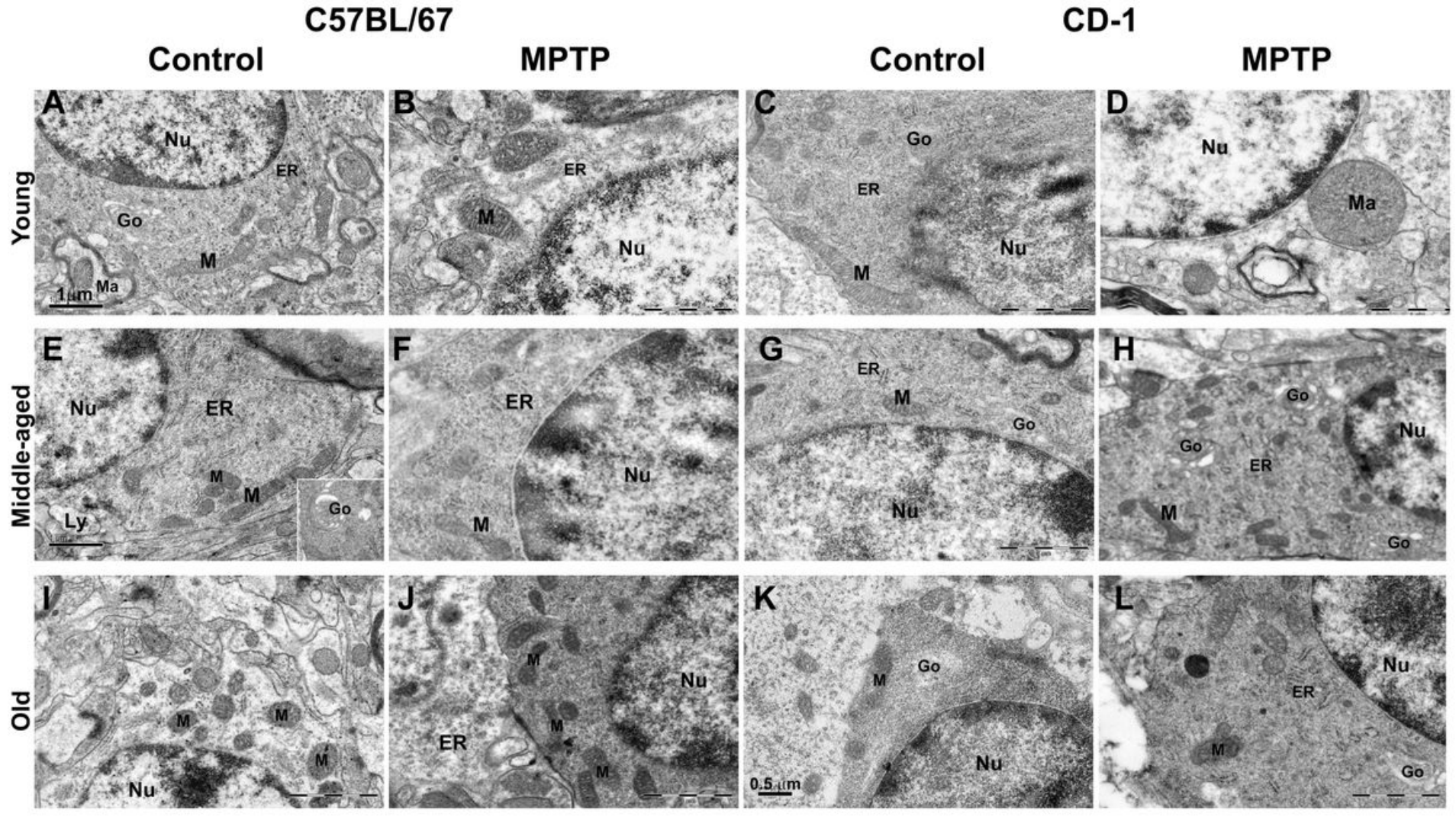

Figure 6

Astrocytic organelles mostly resist age and MPTP induced alterations Representative electron micrographs of astrocytes at different ages and in response to MPTP.Astrocytes had large and euchromatic nucleus. Note the numerous long and tubular cytoplasmic mitochondria in young and middle aged C57BL/6 $\mathrm{J}$ ('M', compare A\&B and E\&F) and spherical ones ('Ma') in the myelinated axons; oval mitochondria in CD-1 cytoplasm (compare C\&D and G\&H). In old C57BL/6J, the mitochondria are smaller (compare I\&K). MPTP caused qualitative increase in mitochondrial numbers and their elongation (Compare I\&J). Golgi saccules were semicircular and dilated in the middle-aged CD-1 injected with MPTP (Go, compare G\&H and K\&L). The scale bar is $1 \mu \mathrm{m}$ for all micrographs except ' $\mathrm{K}$ '. 


\section{Microglia}

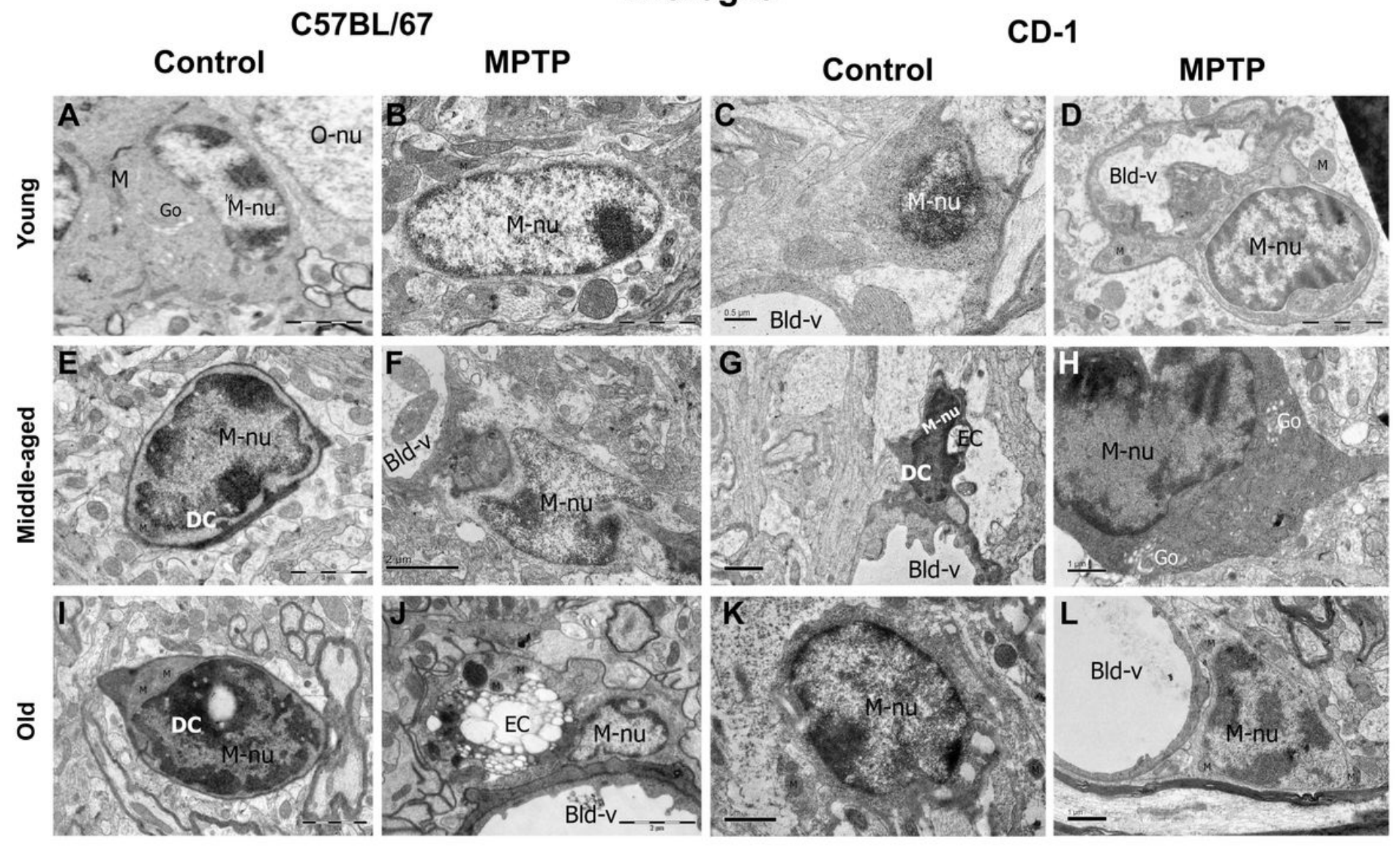

Figure 7

Microglia and Dark cells Representative electron micrographs of microglia at different ages and in response to MPTP. Most microglia are seen near the blood vessels, have bean-shaped nucleus, electron dense cytoplasmand electron dense pockets along the nuclear perimeter. Note the euchromatic nuclei in young (A-D; 'M-nu') and electron dense ones in middle aged and old animals (E-L; 'M-nu'). In the cytoplasm and neighboring tracks of MPTP-injected young C57BL/6J, mitochondria are long and tubular (B, 'M'). Phagocytotic microglia along with the engulfed cells are seen in young CD-1 (D, 'EC') and MPTP-injected old C57BL/6J (J). Amoeboid "dark cells" are seen around the blood vessels in both strains (E, G and I; DC), they have thin rim of cytoplasm containing fewer organelles. The scale bars are specified for each micrograph. 


\section{Vasculature}

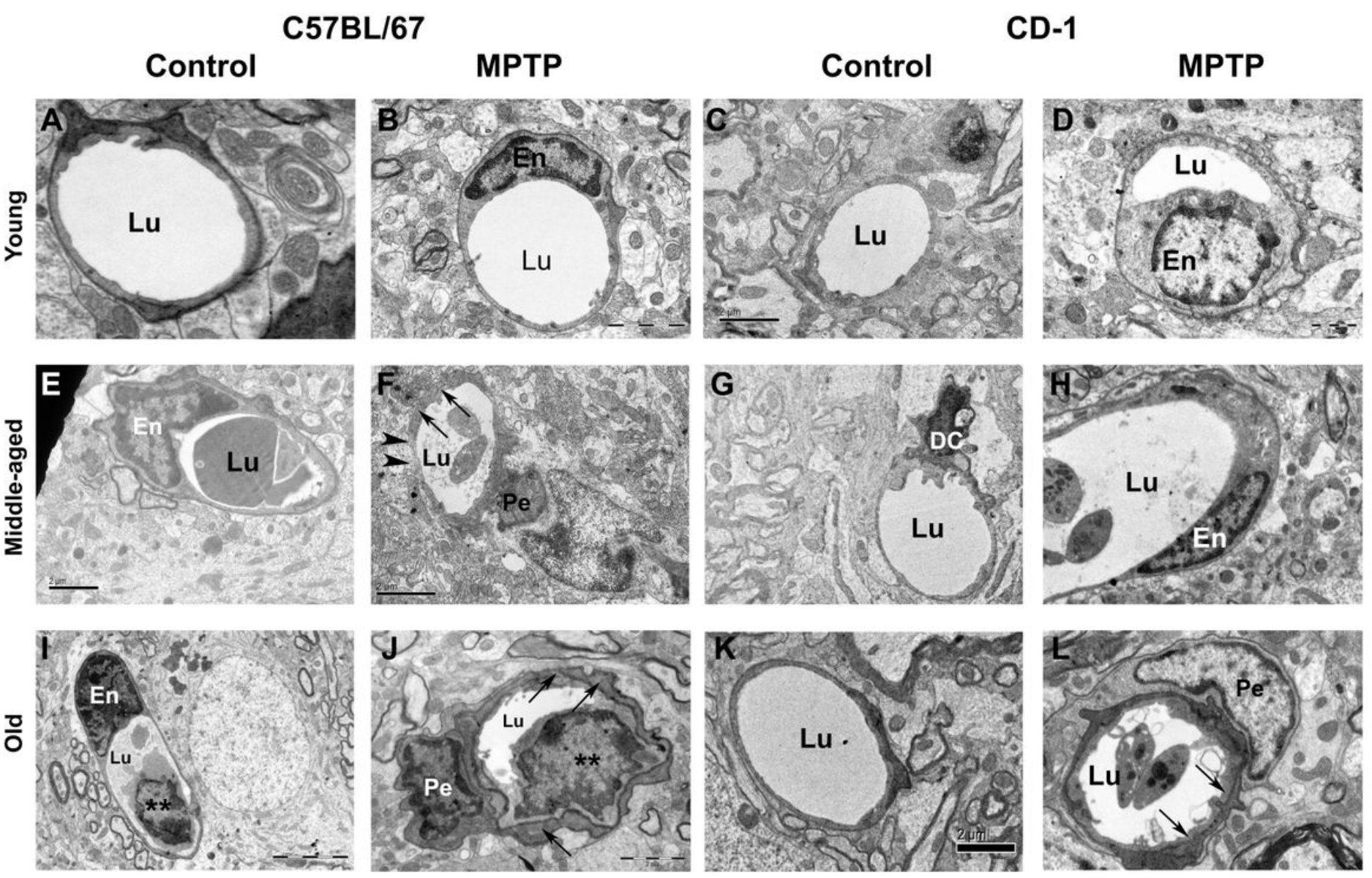

Figure 8

MPTP affects vasculature at middle age in C57BL/6J Representative electron micrographs of blood vessels from different age groups and in response to MPTP. Note the thin basement membrane of the blood vessels in the young (A-D). MPTP injected middle-aged C57BL/6J showa discontinuous membrane suggesting a possible breach, ('F', arrowheads) and thickening of basement membrane in C57BL/6J (arrows, E vs F) but not in CD-1 ( $F$ vs H). Note that at old age, in response to MPTP, the basement membranes of the vessels appear thicker in both strains (arrows, I vs J; K vs L). 


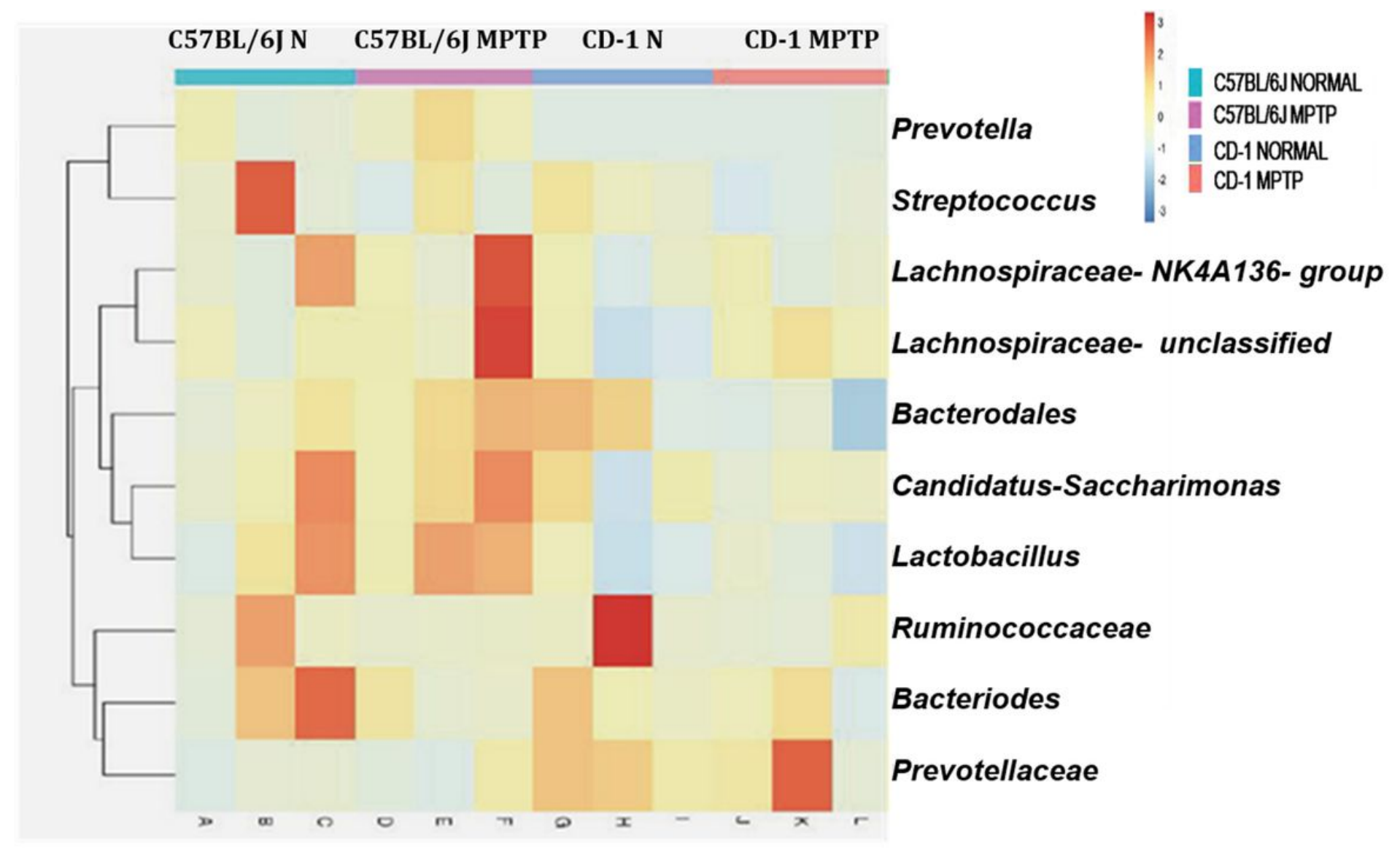

Figure 9

MPTP caused dysbiosis of the fecal microbiome: The heat-map of metagenome-based data analysis, showing differences in the relative abundance of fecal microbiome between C57BL/6J and CD-1 mice under control and MPTP injected situations. Note that the microbes belonging to Streptococcus,

Lachnospiraceae, Bacteriodes, Candidatus, Ruminococcaceaegenus/species are more abundant in C57BL/6J; Bacteriodales, Lactobacillus and Prevotellaceae are more and Prevotellais less in CD-1. Note that MPTP injection increased the Prevotellaceae and Lachnospiraceaespp in CD-1 and Prevotella,

Lachnospiraceae, Bacterodales, and Ruminococcus in C57BL/6J. 